\title{
Time Dependent Lyotropic Chromonic Textures in Microfluidic Confinements
}

\author{
Anshul Sharma (D), Irvine Lian Hao Ong (D) and Anupam Sengupta *(D) \\ Physics of Living Matter, Department of Physics and Materials Science, University of Luxembourg, 162 A, \\ Avenue de la Faïencerie, L-1511 Luxembourg, Luxembourg; anshul.sharma@uni.lu (A.S.); \\ irvine.ong@uni.lu (I.L.H.O.) \\ * Correspondence: anupam.sengupta@uni.lu; Tel.: +352-46-66-44-6733
}

Citation: Sharma, A.; Ong, I.L.H.; Sengupta, A. Time Dependent Lyotropic Chromonic Textures in PDMS-Based Microfluidic Confinements. Crystals 2021, 11, 35 https: / / doi.org/10.3390/ cryst11010035

Received: 10 November 2020 Accepted: 18 December 2020 Published: 30 December 2020

Publisher's Note: MDPI stays neutral with regard to jurisdictional clai$\mathrm{ms}$ in published maps and institutional affiliations.

Copyright: (C) 2021 by the authors. Licensee MDPI, Basel, Switzerland. This article is an open access article distributed under the terms and conditions of the Creative Commons Attribution (CC BY) license (https:// creativecommons.org/licenses/by/ $4.0 /)$.
Abstract: Nematic and columnar phases of lyotropic chromonic liquid crystals (LCLCs) have been long studied for their fundamental and applied prospects in material science and medical diagnostics. LCLC phases represent different self-assembled states of disc-shaped molecules, held together by noncovalent interactions that lead to highly sensitive concentration and temperature dependent properties. Yet, microscale insights into confined LCLCs, specifically in the context of confinement geometry and surface properties, are lacking. Here, we report the emergence of time dependent textures in static disodium cromoglycate (DSCG) solutions, confined in PDMS-based microfluidic devices. We use a combination of soft lithography, surface characterization, and polarized optical imaging to generate and analyze the confinement-induced LCLC textures and demonstrate that over time, herringbone and spherulite textures emerge due to spontaneous nematic $(\mathrm{N})$ to columnar M-phase transition, propagating from the LCLC-PDMS interface into the LCLC bulk. By varying the confinement geometry, anchoring conditions, and the initial DSCG concentration, we can systematically tune the temporal dynamics of the $\mathrm{N}$ - to M-phase transition and textural behavior of the confined LCLC. Overall, the time taken to change from nematic to the characteristic M-phase textures decreased as the confinement aspect ratio (width/depth) increased. For a given aspect ratio, the transition to the M-phase was generally faster in degenerate planar confinements, relative to the transition in homeotropic confinements. Since the static molecular states register the initial conditions for LC flows, the time dependent textures reported here suggest that the surface and confinement effects-even under static conditions-could be central in understanding the flow behavior of LCLCs and the associated transport properties of this versatile material.

Keywords: lyotropic chromonic liquid crystals; microfluidics; surface anchoring; phase transition; herringbone; spherulite; textures

\section{Introduction}

Lyotropic chromonic liquid crystals (LCLCs) are a class of lyotropic liquid crystals (LCs) that are formed by anisotropic assemblies of water-soluble disc-shaped molecules which have an aromatic core surrounded by ionic groups. Most commonly used chromonic LCs are sunset yellow (SSY), an azo dye used as a food additive, and disodium cromoglycate (DSCG), an anti-asthmatic drug. Unlike lyotropic LCs, LCLCs do not form micelles; rather, they stack up as linear aggregates, held together by non-covalent interactions which lead to self-assembled nematic $(\mathrm{N})$ phase or columnar (M-phase) with a hexagonal arrangement [1-4]. In both the $\mathrm{N}$ - phase and the M-phase, the director is parallel to the columnar axis of the stacks, as was revealed by X-ray studies. The nematic phase consists of short columnar stacks of DSCG molecules and appears at room temperature for low DSCG concentrations, whereas with the increase in DSCG concentration, the stacks assemble into a two-dimensional hexagonal array of extended columns in the M-phase [5-7], archetypal herringbone and spherulite textures spanning a wide range of concentration and temperature conditions. The weak interaction forces underpinning the self-assembled 
phases render LCLCs highly responsive to external stimuli (temperature, concentration, $\mathrm{pH}$, ionic content, etc.) and geometric constraints [1-4], thus bestowing distinctive properties such as negative birefringence and a large difference in elastic constants. Owing to the biocompatibility and anisotropic properties, LCLCs have been explored in biological applications such as for drug delivery [8], optical biosensors [9], as well in technological applications such as organic electronics and optical components [10]. Lately, LCLCs have also been explored as host systems for active bacterial systems [11,12].

The challenges involved with the alignment of LCLCs hinder its practical applications. Various techniques from traditional LC alignment methods such as rubbed polyimide [13], external fields [14], oblique evaporation [15], self-assembled monolayers [16], etc., to sophisticated and complex methods such as graphene deposition [17], sputter lithography [18], photo-patterning [19], and plasmonic photopatterning of the liquid crystal polymer network [20] have been explored to achieve uniform alignment. Recent studies by Peng et al. and Taras et al. showed that the photopatterned orientation of LCLC can be used to control the flow of active matter, which can be predesigned to streamline the chaotic movements of swimming bacteria [21,22]. These works demonstrate that precise control of LCLC alignment can be used to control the orientation of active matter and has potential in microcargo delivery and soft microrobotics. However, to align DSCG perpendicular to the surface, only a few techniques such as silanes [23], hydrophobic polymers [24], graphene [17], and parylene [25] have been successful. Recently, Guo and coworkers showed the use of two photon laser writing techniques to generate threedimensional topographical structures that can be used to uniformly and precisely control the alignment of LCLCs [26] (Figure 1).

In the nematic phase, LCLCs are known to have an unusually low twist-elastic modulus $[27,28]$ and large saddle-splay modulus that breaks the reflection symmetry and creates several new chiral director configurations when subjected to microscale confinements. Recently, a few research groups have studied the confinement induced reflection symmetry breaking and new chiral director configurations [25,28-30] of LCLCs. The chiral symmetry breaking for spherical droplets (tactoids) formed by both DSCG and SSY solutions of LCLCs have been reported [31,32]. Jeong and coworkers reported the formation of hexagonally faceted droplets when the system undergoes the transition from the nematicto columnar phase. When LCLCs are confined in glass capillaries with homeotropic boundary conditions, twisted escaped radial and twisted planar polar configurations have been reported. A third chiral configuration, the escaped twist configuration, was observed in capillaries with degenerate planar boundary conditions [28,30]. Dietrich et al. recently reported the same chiral twisted escaped radial configuration in the nematic phase of disc-like micelles of non-chromonic and achiral lyotropic LCs. A recent study showed that reflection symmetry breaking under confinement is not limited to LCLCs and is suggested to be a general phenomenon exhibited by the nematic phase formed by supramolecular aggregates [33]. On the other hand, Baza and coworkers explored the dynamics of the rheological properties of LCLCs under shear flow and showed that DSCG exhibits tumbling characteristics similar to LC polymers [34].

Despite continued efforts to understand LCLC rheological properties, motivated by both fundamental and applied interests, we still lack a mechanistic insight into the LCLC hydrodynamics at microscales - a universal variable in medical, industrial, and biological settings. With the advancement in soft lithography techniques that have enabled the precise design and modulation of microfluidic geometry, pressure, and boundary conditions, both biological and material technology have seen major advancements over the last decades [35]. Recent progress in liquid crystal microfluidics has demonstrated how hydrodynamics, in combination with surface anchoring and confinement, can be harnessed to generate tunable flow and topological structures with potential for novel applications. The works of Sengupta et al. [36-38] and others [39] have shown that by precisely tuning the flow of nematic LCs, anchoring conditions at channel walls, and confinement conditions in microchannels, interesting phenomenon such as defect-mediated 
flow at a low Reynolds number [40], a non-Poiseuille flow profile [41], hydrodynamic cavitation [42], and crosstalk between the topological fields [43] are observed. The works on nematic LCs in microconfinement have revealed significant insight into the interplay of topological defects with microflows of nematic LCs, with potential applications in microcargo systems [44,45], optofluidic systems [46-48], and fluidic resistance circuits [49]. Based on these works, recently, flow-induced deformations have also been studied in chiral nematic LCs [50,51] and blue phases. Spontaneous tunable flow structures [41,52] were given a second look by Čopar and coworkers [53], revealing a hidden non-equilibrium chiral nematic state that could be stabilized by tuning microchannel dimensions and the driving pressure.

a

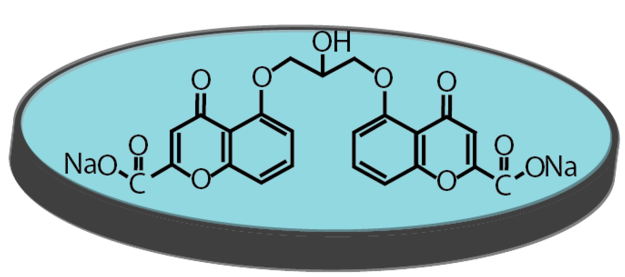

b

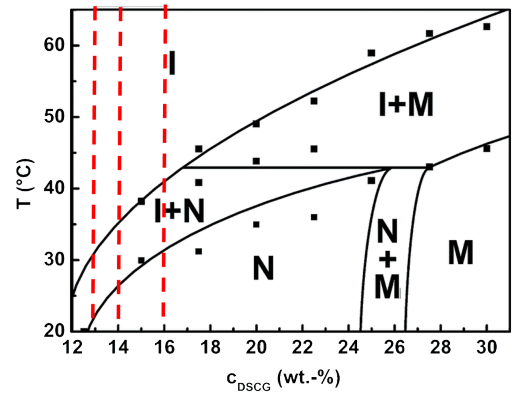

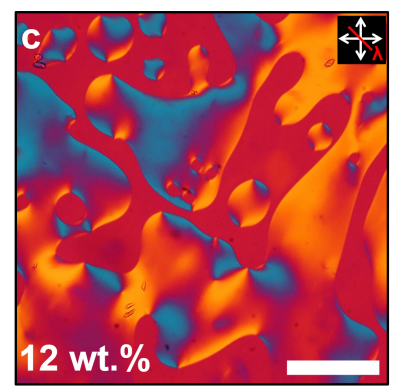
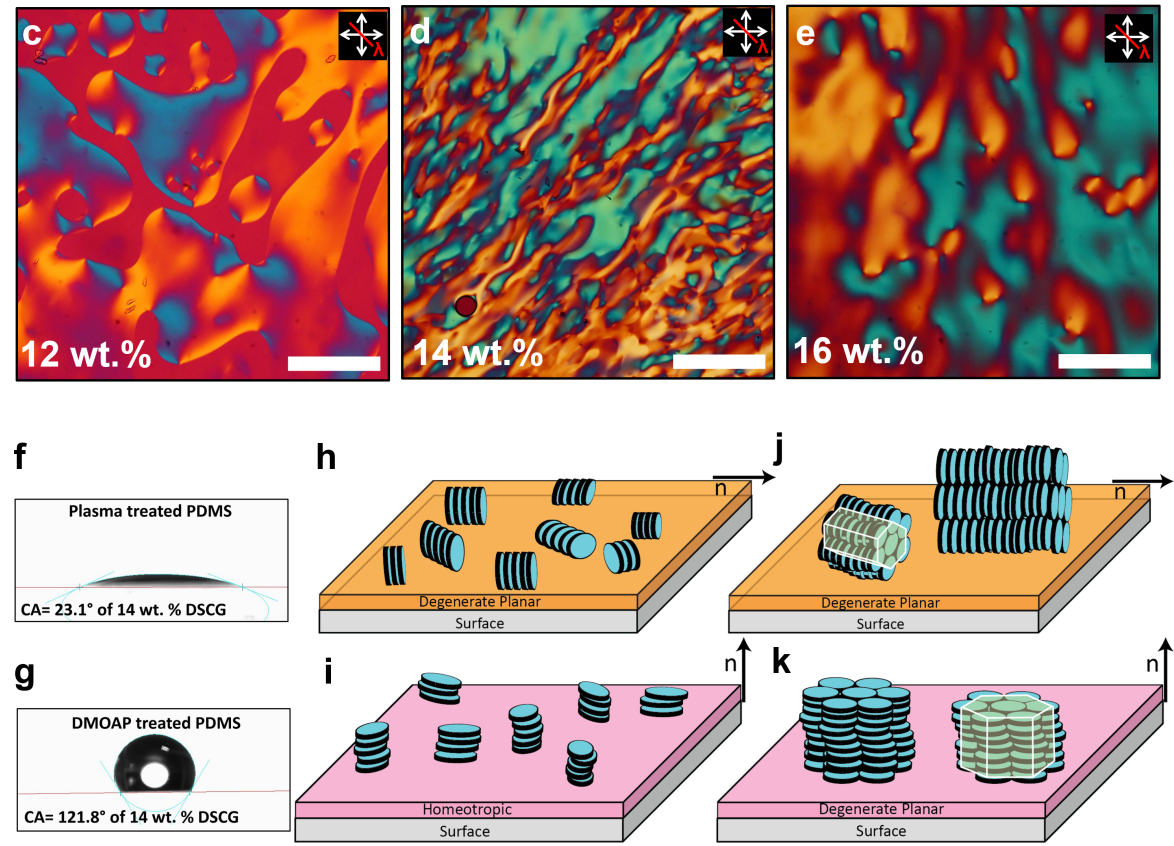

Figure 1. Lyotropic chromonic liquid crystal (LCLC) phases and surface-mediated alignment. (a) Molecular structure of the disodium cromoglycate (DSCG) molecule. (b) Phase diagram of DSCG in water (highlighted dotted red lines indicate the three concentrations used in the current experiments), reproduced with permission from [54]. POM image of (c) the isotropic-nematic co-existence phase formed by $12 \mathrm{wt}$ \% DSCG solution and (d,e) the Schlieren textures due to the nematic phase formed by $14 \mathrm{wt} . \%$ and $16 \mathrm{wt} . \%$ DSCG at $22{ }^{\circ} \mathrm{C}$. The DSCG solutions, imaged between crossed polarizers with a $\lambda$-retardation plate, were confined within cells made of an untreated glass slide and a cover slip, scale: $200 \mu \mathrm{m}$. $(\mathbf{f}, \mathbf{g})$ show the contact angles of the $14 \mathrm{wt} . \%$ DSCG with plasma- and DMOAP-treated PDMS surfaces, respectively. Schematic representation of the LCLCs: $(\mathbf{h}, \mathbf{i})$ for the nematic and $(\mathbf{j}, \mathbf{k})$ the columnar phase in degenerate planar alignment (edge-on arranged molecules) and homeotropic alignment (face-on arranged molecules), respectively.

The behavior of LCLCs in strict microfluidic environments still awaits exploration, with multiple key variables (that determine their interactions) yet to be uncovered and understood. As microfluidic environments offer distinct interplays between the surface, confinement, and viscous parameters, one expects rich and unique interactions 
emerging at the LCLC-microfluidic interfaces. By substituting thermotropic nematic LCs with LCLCs within microfluidic confinements, the authors observed a wealth of novel phenomena-under both static and dynamic conditions-which warrant fundamental investigations. Building on our insights from experiments on thermotropic LCs in strictly microfluidic confinements, here we continue with state-of-the-art soft lithography techniques to fabricate microchannels of different dimensions and modify the surface anchoring conditions on the channel walls. It is known from the study of thermotropic LCs in microfluidic environments that the initial conditions for flow-induced director response are defined by the static (no flow) conditions [52]. With a vision to comprehensively understand the flow-confinement-anchoring couplings in LCLCs confined within microchannels, here we analyze the fundamental no flow state of LCLCs within microfluidic environments. Specifically, we characterize how the LCLC self-organizes within microscale confinements as a function of three fundamental variables: (i) channel dimensions, (ii) anchoring conditions, and (iii) LCLC concentration. In doing so, we uncover a fourth factor-the experimental timescale - which could play an important role in establishing the static director configurations within PDMS-glass microchannels. Our results reveal that the set of four variables is fundamental in determining the outcome of flow experiments (flow results will be discussed elsewhere) and thus need to be taken into account when designing LCLC microfluidic experiments. In our first attempt to bridge the gap between LCLC dynamics and microfluidics, here we will focus on recent results on the temporal dynamics of LCLC textures formed by static DSCG solutions confined in PDMS-glass microchannels, spanning different aspect ratios $(A R=$ width $/$ depth $=w / d)$ and anchoring conditions. The basic structure of DSCG along with the stack configurations representing different phases are shown in Figure 1.

\section{Materials and Methods}

Using a combination of precision microfluidics and polarization microscopy, we characterized the concentration dependent static LCLC textures. We compared three concentrations of DSCG and characterized the LCLC optical textures as a function of anchoring conditions and channel aspect ratios, using polarizing optical microscopy and image analysis. Our results indicate that the emergence and orientation of the LCLC micro-domains are highly sensitive to the confinement length scales and surface anchoring conditions. Additionally, a highlight of this work is the spontaneous nematic to columnar (M) phase transition in PDMS-glass microchannels, as a function of time (under steady state temperature conditions). The time of the appearance of the $\mathrm{M}$-phase shows a strong dependence on the channel geometry (width and depth) and surface anchoring (degenerate planar vs. homeotropic), indicating the fundamental role played by the properties of the confining surfaces (specifically, PDMS in this case) that need quantitative discussions detailed in [55]. All our experiments were conducted in multiple replicates (a minimum of 3 ) to ensure the reproducibility of the results.

\subsection{Materials and Solution Preparation}

Disodium cromoglycate and dimethyloctadecyl [3-(trimethoxysilyl)propyl] ammonium chloride solution (DMOAP) were purchased from Sigma-Aldrich. 4-cyano-4-pentyl-1, 10-biphenyl (5CB) was procured from Synthon Chemicals and used as received. All the solvents used were of GC purity ( $\geq 99.8 \%$ ) sourced from Carl-Roth. Solutions with 12, 14, and $16 \mathrm{wt}$ \% of DSCG were prepared in Millipore water, vortexed for $30 \mathrm{~min}$ and stirred at room temperature for $12 \mathrm{~h}$ to obtain a homogeneous solution. The two component epoxy Araldit ${ }^{\circledR}$ Rapid was purchased from Carl-Roth. Glass capillaries were acquired from CM Scientific Ltd. (Vitrocom Vitrotubes ${ }^{\circledR}$ ).

\subsection{Microfluidic Confinement}

The experimental protocol used in the fabrication of PDMS microfluidic channels is based on the setup described previously [40]. Briefly, microchannels were constructed 
using polydimethylsiloxane (PDMS, Sylgard 184, Dow Corning) reliefs and prepared by following the standard soft lithography techniques. The substrates were cleaned thoroughly with isopropanol and dried by placing the surfaces on a hot plate $\left(80^{\circ} \mathrm{C}\right.$ for $\left.30 \mathrm{~min}\right)$. The molded PDMS reliefs were bonded to glass substrates after being exposed to air plasma. We prepared linear channels with a rectangular cross-section. The channel depth (d) was varied between 10 and $40 \mu \mathrm{m}$, while the channel width $(w)$ was varied between 100 and $345 \mu \mathrm{m}$. The distance between the inlet and the outlet port was set to $20 \mathrm{~mm}$, defining the length $(l)$ of the channel. Different regions along the channel length were observed under the microscope to estimate the uniformity of functionalization. At both ends of the microchannel, cylindrical holes with a $1.5 \mathrm{~mm}$ diameter were punched through the PDMS to provide the housings for the tubings. Two Teflon (PTFE) tubes with a $0.5 \mathrm{~mm}$ inner diameter and a $1.58 \mathrm{~mm}$ outer diameter were inserted into each of the housings and served as connectors for the inlet-to-source and outlet-to-sink, respectively. For glass devices, hollow rectangle capillaries of dimensions (depth $\times$ width) $10 \times 100 \pm 10 \% \mu \mathrm{m}$, $40 \times 400 \pm 10 \% \mu \mathrm{m}$, and $100 \times 1000 \pm 10 \% \mu \mathrm{m}$ were used to study the texture development of LCLCs. Glass capillaries were either used as such or treated in order to obtain suitable surface anchoring and then filled with the liquid crystal.

\subsection{Surface Anchoring and Boundary Conditions}

Microfluidic devices were functionalized for investigating LCLC textures within microchannels and glass capillaries possessing two distinct surface anchoring conditions: degenerate planar and homeotropic. Specific anchoring states within the microfluidic devices were achieved using a combination of different physical and chemical methods. We applied well studied plasma exposure (inducing degenerate planar anchoring) and DMOAP treatment (homeotropic) for surface functionalization of the PDMS-glass microchannels and glass-only devices [52,56]. The anchoring conditions were validated using thermotropic nematic 5CB as our control sample, for which detailed studies exist [57].

The degenerate planar surface anchoring was obtained after treatment of the PDMS surface by air plasma. Experiments under degenerate planar alignment conditions were conducted under freshly treated channels only. For homeotropic alignment, the channels were first filled with $0.4 \mathrm{wt} . \%$ aqueous solution of DMOAP and then dried in an oven at $80{ }^{\circ} \mathrm{C}$ for $2 \mathrm{~h}$ to render both the PDMS and glass surfaces hydrophobic, inducing perpendicular (homeotropic) boundary conditions. In order to achieve homeotropic anchoring of the director at the LC-glass interface, the glass capillaries were filled with a $0.4 \mathrm{wt} . \%$ aqueous solution of DMOAP, and the solvent was allowed to evaporate before the capillary was filled with the liquid crystal. The anchoring inside the capillaries was characterized through the shape of the meniscus of water and nematic $5 \mathrm{CB}$, relative to the shape inside native capillaries, as shown in Figure A2.

\subsection{Filling the Microfluidic Devices}

Glass-PDMS and glass capillaries were filled above the isotropic-nematic transition temperature of the LCLC solution for a given concentration of DSCG $(12,14$, or $16 \mathrm{wt} . \%)$ at $45{ }^{\circ} \mathrm{C}$, either with a syringe and needle or capillary filling, respectively. Typically, a microchannel kept at $45{ }^{\circ} \mathrm{C}$ was filled with a syringe $(3 \mathrm{~mL})$ with a beveled tip needle (23 gauge, $25 \mathrm{~mm}$ long, $0.6 \mathrm{~mm}$ outer diameter), to which a PTFE tube (0.5 $\mathrm{mm}$ inner diameter and $1.58 \mathrm{~mm}$ outer diameter) was connected. The other end of the PTFE tube was inserted into the microchannel. After filling, the microchannel was sealed with epoxy glue, to avoid flow-induced effects (e.g., alignment) and, crucially, to prevent water evaporation, which could change the DSCG concentration. Thereafter, microchannels were slowly cooled down to $22{ }^{\circ} \mathrm{C}$ with a ramp rate of $1.5^{\circ} \mathrm{C}$ per minute. The capillaries were filled with $14 \mathrm{wt} . \%$ DSCG solution by capillarity at $45^{\circ} \mathrm{C}$ and then immediately sealed with epoxy to prevent water evaporation and to avoid flow-induced effects, then allowed to slowly cool down to $22^{\circ} \mathrm{C}$ with a ramp rate of $1.5^{\circ} \mathrm{C}$ per minute. 


\subsection{Optical Microscopy and Image Acquisition}

The director orientation of LCLCs in the PDMS-microchannels and glass capillaries was determined using a polarized light microscope (Eclipse LV100N-POL, Nikon), equipped with $\times 5, \times 10, \times 20$, and $\times 40$ objectives and with a Linkam (PE120) heating/cooling stage. The samples were observed between crossed polarizers in the transmission mode using monochromatic light and several polarizer configurations. Additionally, the channels were oriented at $45^{\circ}$ with respect to the polarizers and with and without the insertion of a $530 \mathrm{~nm}$ phase-retardation plate. The images $(6000 \times 4000$ resolution) and videos (1920 × 1080 resolution) were acquired using a digital Canon EOS77D camera, and videos were recorded in full HD color at a frame rate of 25 frames per second. The image and video analysis was performed by using the tools Image ${ }^{\circledR}$ and VideoMach ${ }^{\circledR}$, respectively.

\section{Results and Discussion}

The goal of this work is to study the equilibrium textures LCLC under strict microfluidic confinements possessing distinct surface anchoring conditions and channel dimensions. Figure 2 shows the experimental variables considered here: channel dimension, anchoring conditions, and experimental timeline. For experiments conducted with PDMS-glass microchannels, all four walls possessed either a degenerate planar anchoring or homeotropic anchoring. From hereon, the results presented will focus on the concentration of DSCG of $14 \mathrm{wt} . \%$, as our control case. Over the following sections, we present the dynamics of the emergence of the LCLC textures due to the DSCG solution. Based on the initial observations, a number of key questions-on the emergence of the dynamic and equilibrium LCLC textures-arise, the answers to which could identify specific roles of surface anchoring, confinement, and LCLC concentrations. Below, we present a step-by-step, detailed report on the emergent textures as a function of the aforesaid factors.
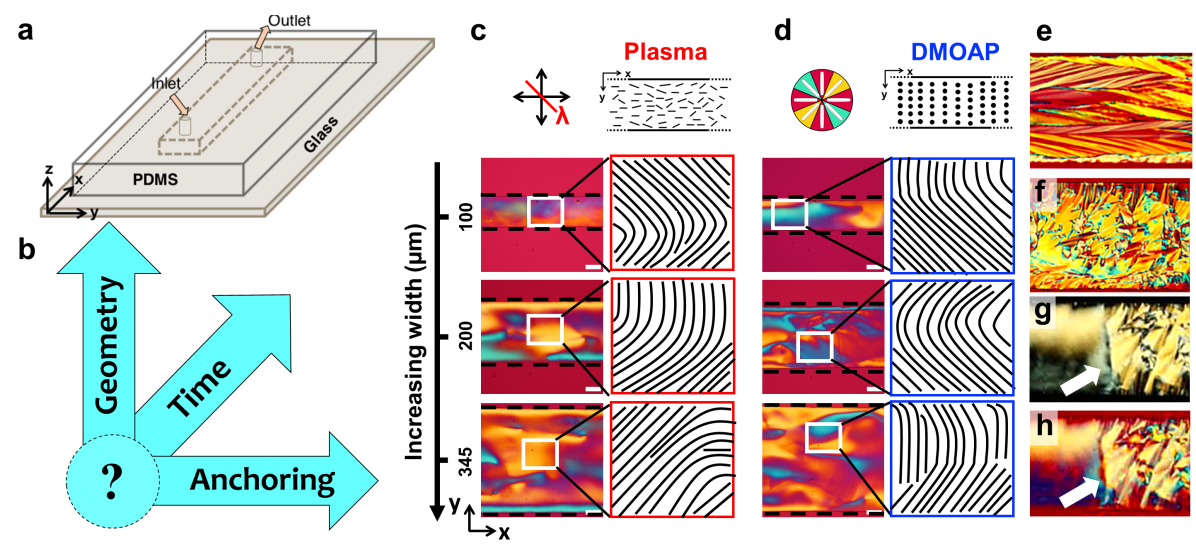

Figure 2. Experimental scheme and parameters considered in this work. (a) PDMS-glass microfluidic devices were fabricated using the soft lithography technique, whereby the channel geometry and anchoring conditions could be altered systematically. (b) In a nutshell, the key variables considered in this study: channel geometry, anchoring conditions, and the time elapsed since the isotropic-nematic transition. (c) PDMS-glass microfluidic devices possessed degenerate planar anchoring with dimensions (from top): (100 $\mu \mathrm{m}$ wide, $10 \mu \mathrm{m}$ deep), (200 $\mu \mathrm{m}$ wide, $10 \mu \mathrm{m}$ deep), and (345 $\mu \mathrm{m}$ wide, $10 \mu \mathrm{m}$ deep), respectively; and (d) presents the corresponding textures within channels treated for homeotropic anchoring. Microfluidic textures exhibited by the $14 \mathrm{wt}$ \% DSCG, shortly after the isotropic-nematic phase transition, are imaged between crossed polarizers with a $\lambda$-retardation plate. The two-dimensional integrated director field of the LCLC in the nematic phase (sketched alongside) indicates the planar director configuration. Overall, no significant difference is observed between the two anchoring conditions during the short time span elapsed (order of a few minutes) after the isotropic-nematic transition. Scale bars: $50 \mu \mathrm{m}$. (e-h) Polarized optical images represent stable LCLC M-phase textures at longer timescales (of the order of hours) within a $200 \mu \mathrm{m}$ wide, $10 \mu \mathrm{m}$ deep channel. Degenerate planar anchoring induces herringbone texture (e), and homeotropic anchoring induces spherulite texture (f), as observed between crossed polarizers with the $\lambda$-retardation plate. Panels $(\mathbf{g}, \mathbf{h})$ capture the transient nematic to M-phase interface, shown here using white arrow, between crossed polarizers (without and with the $\lambda$-retardation plate, respectively). 


\subsection{LCLC Microfluidic Textures Due to Degenerate Planar Anchoring}

As shown in Figure 2a-f, POM imaging was used to characterize the developing director orientation within the microchannels. To create the degenerate planar anchoring, we employed plasma treatment (discussed previously) as a way to modify the microchannel surfaces to align the nematic phase exhibited by $14 \mathrm{wt} . \%$ DSCG. The plasma-treated microchannel was filled with DSCG, sealed, and cooled to $22^{\circ} \mathrm{C}$ with a ramp rate of $1.5^{\circ} \mathrm{C}$ per minute. In order to perform preliminary optical characterization of the textures, we scanned the entire channel at a lower magnification $(\times 5)$, followed by images from selected areas (that best represented the overall director profile) taken at a higher magnification $(\times 20)$, presented in Figure 2a-c. The corresponding textures in the homeotropic channels are shown in Figure $2 \mathrm{~d}-\mathrm{f}$, which interestingly, right after the isotropic to nematic phase transition, resemble the degenerate planar textures texture. The anchoring condition was confirmed to be homeotropic by filling in 5CB and comparing the resulting texture with those in our previous works.

Figure 3 captures the time evolution of the DSCG textures within channels possessing degenerate planar anchoring. The dimension of the microchannel was kept fixed, with an $A R \approx 10(w=100 \mu \mathrm{m}, d=10 \mu \mathrm{m})$. Figure 3 (I-II) shows the POM micrographs obtained from a combination of different polarizer orientations, used to deduce the average molecular orientations corresponding to the planar textures. The birefringent domains appear shortly after the sample is cooled down below the isotropic-nematic phase transition temperature and stabilize over a timescale of $60 \mathrm{~min}$. This appears as dark and bright regions between crossed polarizers, which show the formation of randomly oriented planar DSCG domains. The textures were further characterized using a $\lambda$-retardation plate along with the crossed polarizers (II). It may be worthwhile to note here that the DSCG (nematic and M-phase) possesses negative birefringence [58]; thus, in our POM micrographs with $\lambda$-retardation plates, the regions in yellow represent the nematic director orientation parallel to the slow axis of the retardation plate (please see Figure 2 for the reference color wheel). The region, where the director is oriented perpendicular to the slow axis of the retardation plate, appears blue [59]. The color variation can be attributed to the varying LC director orientations of microdomains formed by LCLC stacks in the nematic phase. In order to determine the stability of the planar textures, we checked the texture evolution every $15 \mathrm{~min}$ after the ramp had finished, for at least $60 \mathrm{~min}$. Our observations reveal that initially (right after transition, $\mathrm{t}=0 \mathrm{~min}$ ), the DSCG solution has degenerate planar orientations. Different parts of the microfluidic channel were monitored to ascertain the homogeneity of the textures along the channel length. For the region of interest presented here, the planar nematic textures were found to be stable and homogeneous for about $60 \mathrm{~min}$. 


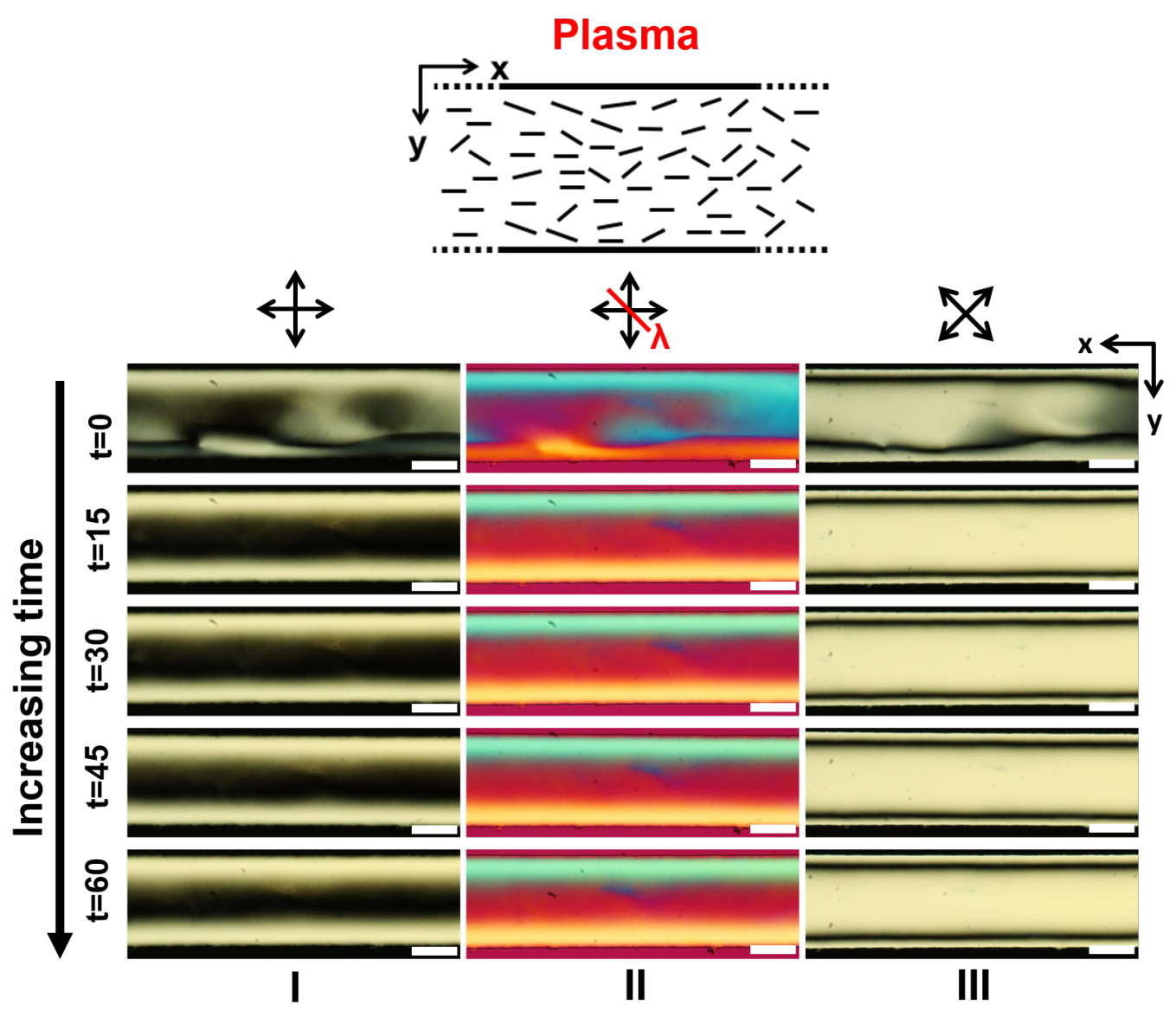

Figure 3. Nematic textures of 14 wt.\% DSCG within microchannels possessing degenerate planar anchoring. Polarizing optical micrographs show the time evolution of the planar nematic textures in $14 \mathrm{wt}$ \% DSCG after the isotropicnematic transition, observed over a duration of $60 \mathrm{~min}$, between: (I) crossed polarizers; (II) crossed polarizers and the $\lambda$-retardation plate; (III) crossed polarizers with $45^{\circ}$ rotation. Here, time $(\mathrm{t})$ is in minutes after the first appearance of the nematic domains. The microchannels were $100 \mu \mathrm{m}$ wide and $10 \mu \mathrm{m}$ deep, scale bar: $50 \mu \mathrm{m}$.

Interestingly, within similar timescales, isolated microdomains of the M-phase (visualized as non-planar static nematic textures) appeared, as shown in (Figure 4). Starting from a degenerate alignment, such textures grew and propagated over time, ultimately stabilizing into a herringbone configuration, a classical M-phase texture observed in LCLC materials. Figures 4, 5 and A18 (I) present different manifestations of the M-phase observed in our experiments. Tone et al. reported a similar degenerate planar texture for a $13 \mathrm{wt} . \%$ DSCG solution in polylysine-coated LC glass cell [15]. It was also observed that the degenerate planar texture evolved to a ribbon structure, a texture corresponding to the coexistence of the $\mathrm{M}$ and isotropic phase. As there is no preferential alignment of the director along any direction, the DSCG stacks orient randomly, forming a multi-domain structure of different orientations of the director. However, the timescale over which the planar textures transition from nematic to herringbone (M-phase) depends on the channel aspect ratio and the DSCG concentration, as discussed in Section 3.3. 


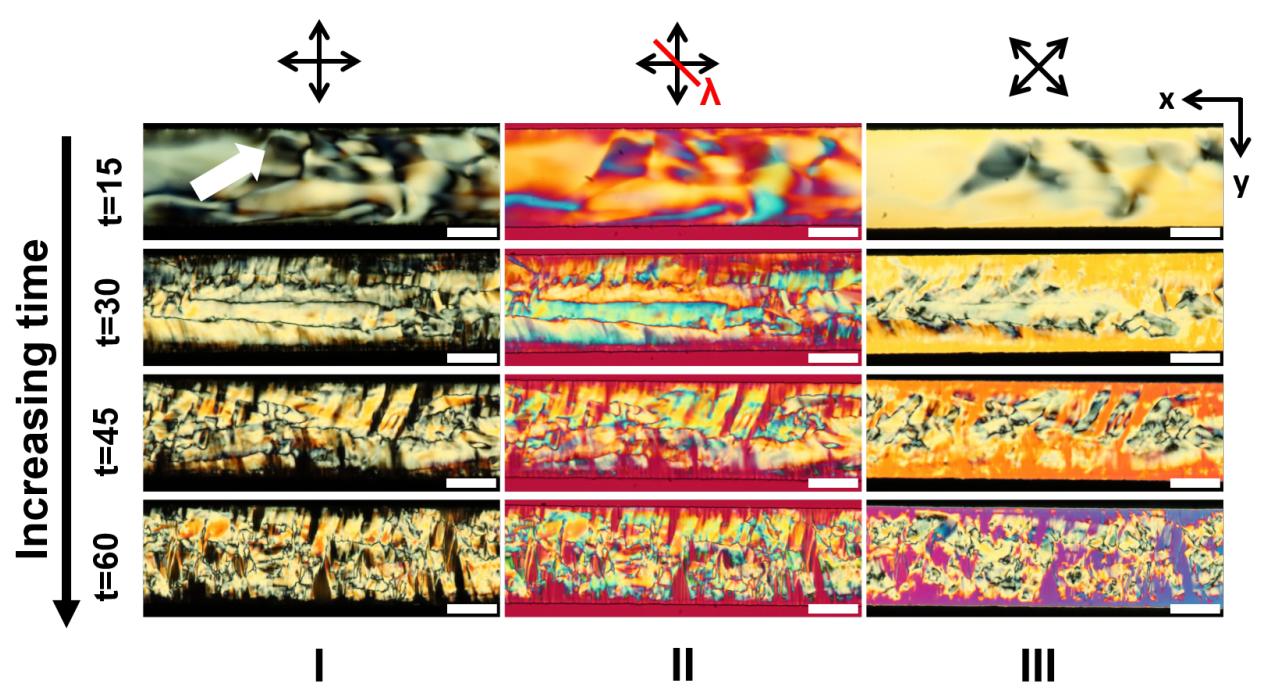

Figure 4. Appearance of the herringbone microfluidic texture due to the formation of the M-phase in degenerate planar microchannels. (I)-(III) present the formation of the characteristic herringbone texture (in the M-phase) in $14 \mathrm{wt} . \%$ DSCG, seen here on the right half of the micrographs (the appearance of herringbone can be seen at $t=15 \mathrm{~min}$, on the right boundary of the image, shown here using a white arrow head). The micrographs were captured using different orientations of the polarizers relative to the channel, with or without the $\lambda$-retardation plate, as described in Figure 3 . Here, time (t) is in minutes after the first appearance of the nematic domains. Microchannels were $100 \mu \mathrm{m}$ wide and $10 \mu \mathrm{m}$ deep, scale bar: $50 \mu \mathrm{m}$.
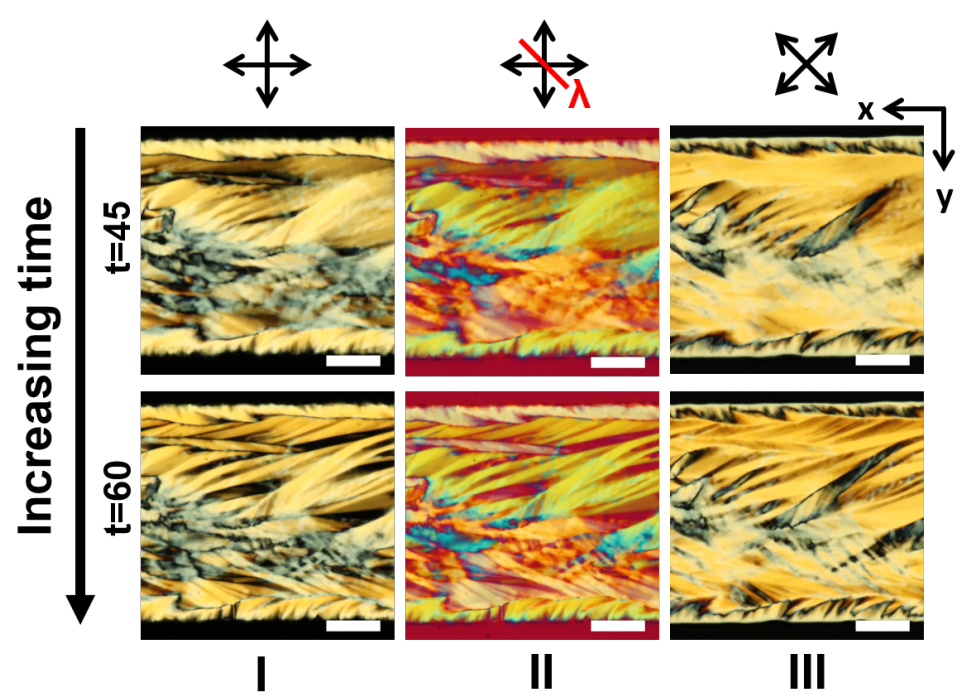

Figure 5. Stabilization of the herringbone texture in a (200 $\mu \mathrm{m}$ wide, $10 \mu \mathrm{m}$ deep) microchannel with degenerate planar anchoring. The well developed $\mathrm{M}$-phase texture (at $\mathrm{t}=45 \mathrm{~min}$ ) stabilized at around $\mathrm{t}=60 \mathrm{~min}$. Thereafter, no perceptible change in the herringbone texture was observed, consistently across multiple replicates. The images present polarized micrographs under different crossed polarizer configurations, as described in previous images. Globally, the herringbone structure is oriented parallel to the length of the microfluidic device. (I-III) shows the POM micrographs obtained from a combination of different polarizer orientations at the corresponding time. Scale bar: $50 \mu \mathrm{m}$.

\subsection{LCLC Texture in Homeotropic Microchannels}

We explored the surface treatment with silanes as a way to modify the surface of PDMSglass microchannels that support homeotropic anchoring. DMOAP-treated microchannels (prepared freshly) were filled with $14 \mathrm{wt} . \%$ DSCG solution in the isotropic phase, sealed, and then allowed to cool down to $22{ }^{\circ} \mathrm{C}$, maintaining a steady ramp rate of $1.5{ }^{\circ} \mathrm{C}$ per minute, as reported previously for the plasma-treated glass-PDMS microchannels. Figure 6 
presents the LCLC textures of the $14 \mathrm{wt}$.\% DSCG solution within homeotropic PDMSglass microchannels, formed right after the isotropic to nematic transition. The non-zero birefringence patterns suggest that, within the homeotropic channels, the DSCG molecules self-organize into uniformly aligned planar or tilted domains of DSCG columns over the observed time window.

By capturing the evolution of the textures over time, we observe that, initially, the LCLC textures formed within channels with homeotropic anchoring resemble the texture formed within channels with degenerate planar anchoring. We call this as the pseudo planar texture, arising due to the planar or tilted organization of the DSCG discotic stacks in the nematic phase. This is in contrast to the thermotropic nematic textures under homeotropic boundary conditions: here, the polarized light undergoes complete extinction upon passing through the sample, thus appearing completely dark regardless of the polarizer orientation. As observed in the degenerate planar case (previous section), for the homeotropic channels as well, we observe isolated microscale domains of the M-phase nucleation, however at a slower rate than that under the planar conditions. Once formed, the domains propagate through the channel, filling it up completely within about $60 \mathrm{~min}$ (Figure 7).

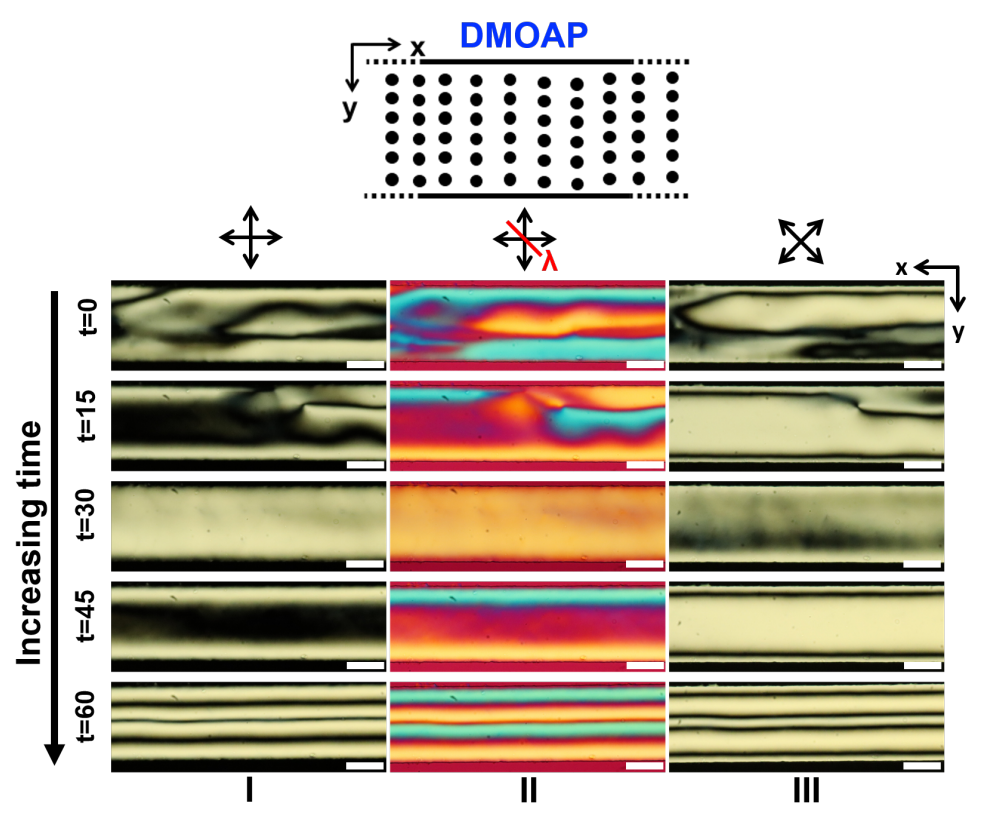

Figure 6. LCLC texture in microchannels possessing homeotropic anchoring. Polarizing optical micrographs capture the time evolution ( $\mathrm{t}=0$ to $60 \mathrm{~min}$ ) of LCLC texture within homeotropic device. The pseudo planar texture arises due to the spontaneous organization of the confined DSCG solution into planar or tilted regions of DSCG stacks. (I,III) are imaged between crossed polarizers with the channel oriented parallel and at $\pi / 4$ to the polarizer, respectively; (II) micrographs imaged between crossed polarizers with an additional $\lambda$-plate. Here, time $(t)$ is in minutes after the first detection of birefringence after the isotropic-nematic transition. Scale bar: $50 \mu \mathrm{m}$.

The DSCG M-phase under homeotropic conditions manifests as the characteristic spherulite texture, whereas within planar microchannels, the M-phase resembles the herringbone texture. These contrasting outcomes-herringbone microfluidic texture in degenerate planar and spherulite texture in homeotropic boundary conditions-suggest that the anchoring conditions play a key role in establishing the equilibrium LCLC textures, in absence of flows, within PDMS-glass microfluidic confinements. Nazarenko and coworkers showed a weak homeotropic anchoring on hydrophobic silane-treated substrates [23], which eventually, at a later time, transitioned from homeotropic to planar anchoring, while Tone et al. reported that use of silane to get homeotropic alignment, which does not work on glass LC cells [24]. From the textural evolution shown in Figure 6, it can be deduced that the emergent pseudo planar DSCG texture is in contrast with the typical homeotropic align- 
ment of thermotropic LCs within similar DMOAP-treated channels (the silane DMOAP induces homeotropic surface anchoring). Furthermore, the areas with homeotropic textures were found to be unstable, and as presented using the time lapse sequence in Figure A22, the texture changes spontaneously from the pseudo planar to the spherulite texture.

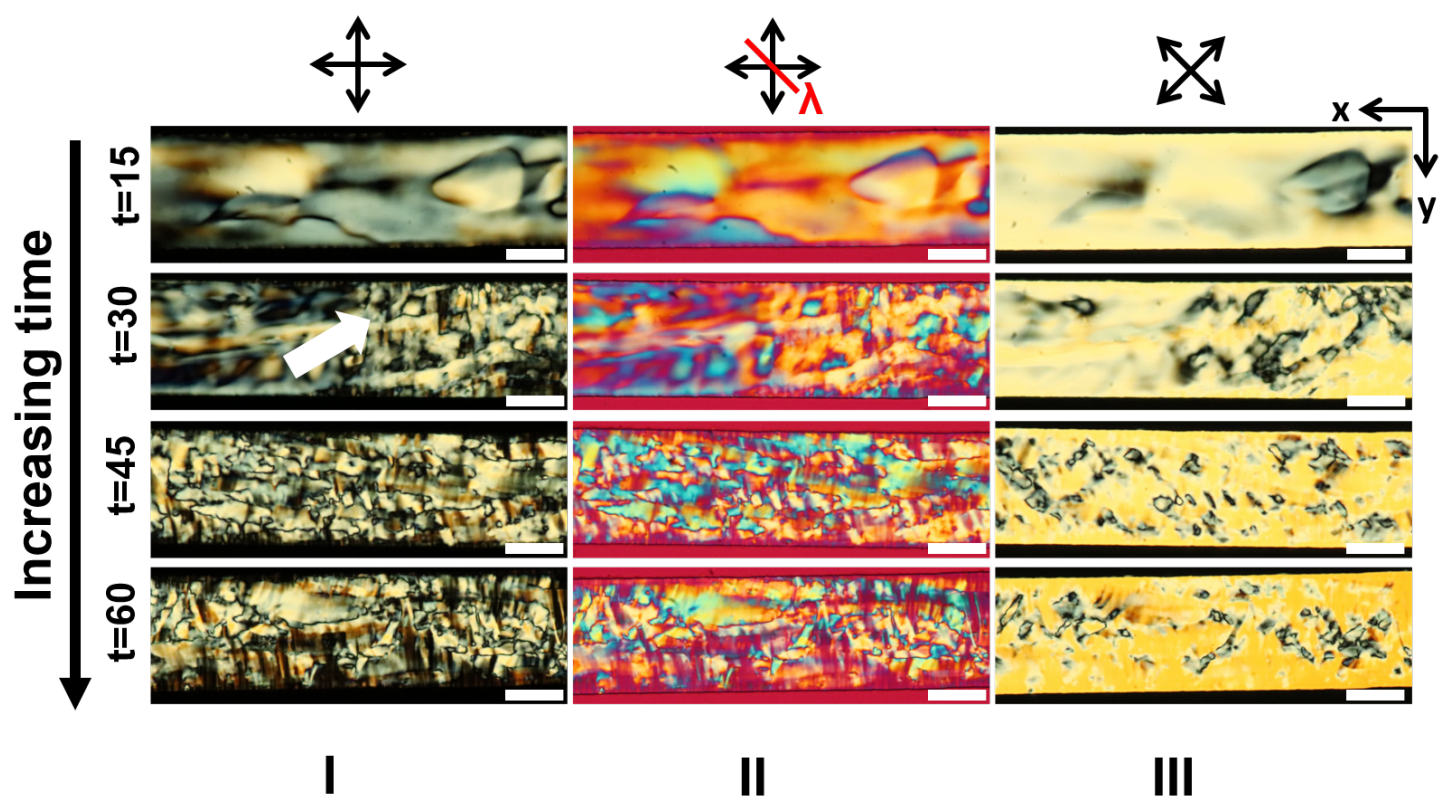

Figure 7. Transition of pseudo planar texture to spherulite texture over time in $14 \mathrm{wt}$ \% DSCG, within microchannels functionalized for homeotropic anchoring. As indicated by the white arrow head, the appearance of the spherulite texture can be seen at $t=30 \mathrm{~min}$, on the right boundary of the image. Polarizing optical micrographs show the top view of the channel from $t=15$ to $60 \mathrm{~min}$. The micrographs were captured using different orientations of the polarizers relative to the channel length, with or without the $\lambda$-retardation plate, as described in Figure 6 . (I-III) shows the POM micrographs obtained from a combination of different polarizer orientations at the corresponding time. Scale bar: $50 \mu \mathrm{m}$.

\subsection{Role of the Channel Dimensions on the Evolution of the LCLC Textures}

The role of microfluidic dimensions was investigated by varying the channel aspect ratio: the ratio between the channel width, $w$, and the channel depth, $d(A R=w / d)$. We studied the texture evolution by systematically varying both the channel width and depth, through the following combinations of $w \times d$ (all dimensions in micrometers): (i) variation of width $($ in $\mu \mathrm{m}): 100 \times 10,200 \times 10$, and $345 \times 10$; and variation of depth $($ in $\mu \mathrm{m}): 100 \times$ $10,100 \times 25$, and $100 \times 40$.

\subsubsection{Role of the Channel Width}

The role of channel width was investigated by studying two additional dimensions: $200 \mu \mathrm{m}$ and $345 \mu \mathrm{m}$, while keeping the depth fixed at $10 \mu \mathrm{m}$. All other experimental conditions and parameters were kept similar to those in the experiments with the $100 \mu \mathrm{m}$ wide channels. As described earlier, the stability of the degenerate planar anchoring was checked every 15 min after the cooling ramp concluded (this coincided with the first appearance of the birefringent domains). For both the $200 \mu \mathrm{m}$ and $345 \mu \mathrm{m}$ wide microchannels, the observations lasted up to $60 \mathrm{~min}$ (or slightly longer, as the case was in a few instances), till the equilibrium LCLC static texture was observed, i.e., no further change in the overall pattern and birefringence. Figure 8 presents the POM micrographs of the $200 \mu \mathrm{m}$ wide, $10 \mu \mathrm{m}$ deep microchannels possessing degenerate anchoring conditions. 


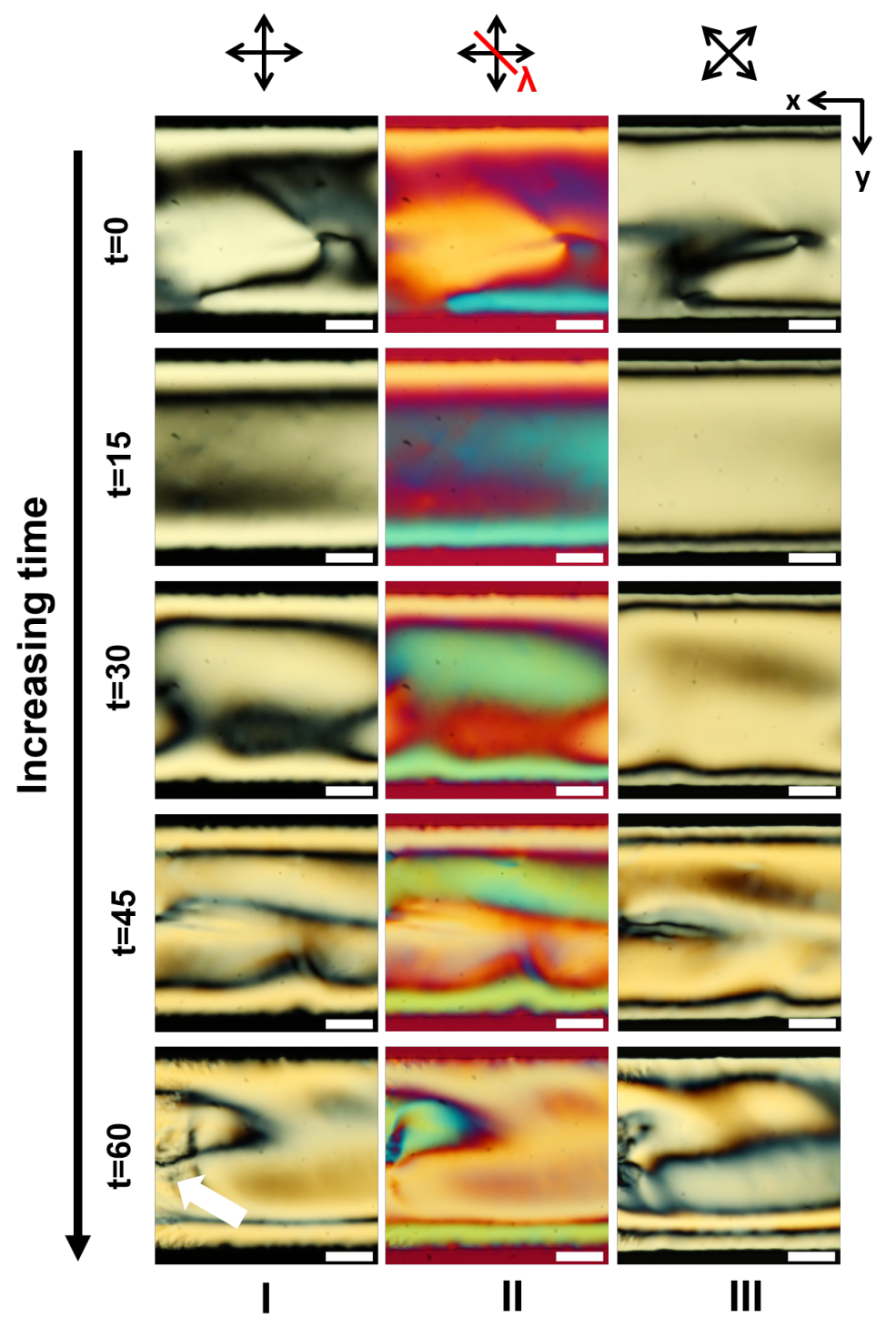

Figure 8. Increasing channel width accelerates the nematic texture to herringbone transition. Evolution of the textures shown as a function of time, within $200 \mu \mathrm{m}$ wide, $10 \mu \mathrm{m}$ deep microchannel possessing degenerate planar anchoring, captured through polarized optical micrographs over $t=0$ to $60 \mathrm{~min}$ (the appearance of the herringbone can be seen at $\mathrm{t}=60 \mathrm{~min}$, on the left boundary of the image, shown here using a white arrow head): (I) between crossed polarizers; (II) between crossed polarizers with the $\lambda$-plate inserted ; (III) similar to I and II, but with the crossed polarizers rotated by $45^{\circ}$. To the left of the Schlieren-herringbone interface (shown by the white arrow), the M-phase texture was well developed (Figure 5). Here, time ( $t$ ) is in minutes after completion of the cooling ramp. Scale bar: $50 \mu \mathrm{m}$.

Initially, we observed the expected planar texture of DSCG due to the degenerate planar anchoring conditions on the channel walls. The planar microdomains, signifying different orientations of the DSCG discotic stacks in the nematic phase, gave way to the nucleation of a more ordered two-dimensional lattice of the M-phase herringbone texture. Starting from a degenerate alignment (Figure 8), the herringbone texture proliferated along the channel length and, over a timescale of about $60 \mathrm{~min}$, covered the entire channel (shown in Figure 5). It might be worth noting that the herringbone texture manifests a global orientation parallel to the length of the microchannel: this suggests that under degenerate planar anchoring conditions, the microfluidic geometry underpins the emergent textural anisotropy of the confined DSCG. Figure A18 (I) captures the variation of the textural anisotropy with the dimensions of microchannels possessing degenerate planar anchoring. 
The herringbone texture was found to be most pronounced within the $200 \mu \mathrm{m} \times 10 \mu \mathrm{m}$ channels.

On increasing the width to $345 \mu \mathrm{m}$, as shown in Figure A3, we observed the textural transition from Schlieren to herringbone texture consistently; however, for wider channels, the transition occurs over a shorter timescale. The overall dependence of the transition time on channel width (and the aspect ratio) is summarized in Figure 9; a physical basis of the same will be reported elsewhere [55].

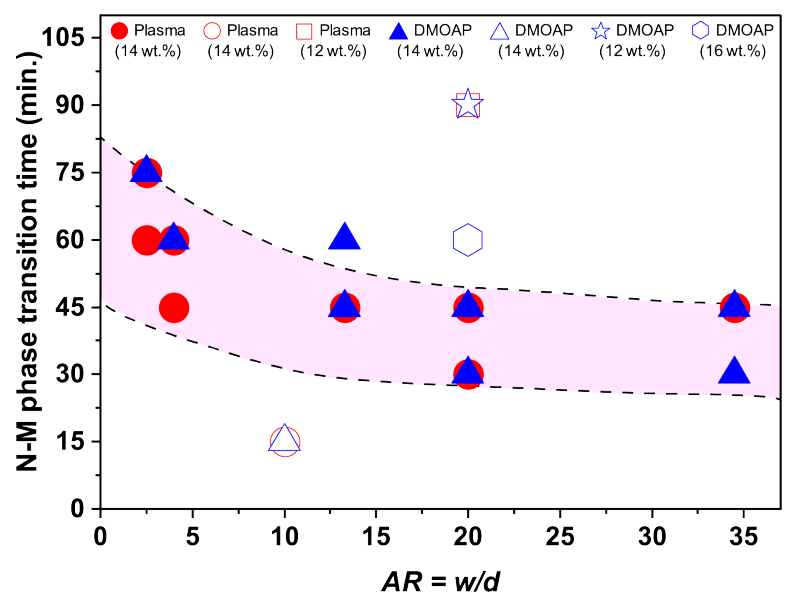

Figure 9. Surface-induced phase transition timescales. The plot summarizes the time dependence of nematic to M-phase textural transitions on the aspect ratio of the microfluidic devices, shown for various DSCG concentrations (12, 14, and $16 \mathrm{wt} . \%)$ and anchoring conditions (degenerate planar vs. homeotropic). Red circles: degenerate planar-14 wt.\%, blue triangle: homeotropic-14 wt.\%, (the empty red circle and empty triangle represent the outliers for $14 \mathrm{wt} . \%$ ), red square: degenerate planar-12 wt. \%, blue star: homeotropic-12 wt.\%, and hexagon: homeotropic-16 wt.\%. The shaded region captures the overall trend of the nematic to $\mathrm{M}$-phase transition time over the confinement aspect ratio. Data points of the individual replicates are shown in the Appendix A, Figure A1. For all the samples, the time reported corresponds to the time at which the M-phase first appears after the cooling ramp ends.

Moving from degenerate planar to homeotropic anchoring conditions, the texture initially appears patchy (Figure A4), with complete extinction of the polarized light, a characteristic signature of homeotropic anchoring. Over a time span of about $15 \mathrm{~min}$, the patchy homeotropic domains gradually transformed into the pseudo planar texture, as shown in Figure A4 for the $200 \mu \mathrm{m} \times 10 \mu \mathrm{m}$ DMOAP-treated microchannel. Finally, the planar texture transforms into the M-phase, over a timescale of $30 \mathrm{~min}$. Apart from the textures observed in area of the microchannel as shown in Figure A4, two other areas of this microchannel were observed: one where the M-phase (Figure A5) first nucleated and the other with a stable homeotropic texture (Figure A6), developed at $t=45 \mathrm{~min}$. For areas where the homeotropic texture developed, on observing between crossed polarizers and the rotation of the sample by $45^{\circ}$, the intensity of transmission light remained low. This area with homeotropic alignment further propagated in the microchannel until $t=60 \mathrm{~min}$, but eventually changed to a degenerate planar texture. At another part of the microchannel where the M-phase appeared at $t=30 \mathrm{~min}$ (Figure 7), the observed area completely changed to the M-phase after $45 \mathrm{~min}$. On changing the channel width further to $345 \mu \mathrm{m}$ (Figures A7 and A8), qualitatively, we observed the same trend: regions of low intensity of transmitted light were first observed, which then transformed into pseudo planar (within $t=15 \mathrm{~min}$ ), which eventually changed to the M-phase at $t=45 \mathrm{~min}$. It may be worthwhile to repeat here that, under homeotropic boundary conditions, the M-phase is distinct from that within degenerate planar microchannels: in homeotropic channels, the spherulite texture stretches transversely across the channel width, whereas within the degenerate planar channels, the herringbone structure organizes along the channel 
length (Figure A18). Taken together, these observations indicate that the channel geometry and surface anchoring, together, play a central role in the development of the static LCLC textures within PDMS-glass devices. In the next section, we report the effect of the channel depth on the textural attributes.

\subsubsection{Role of the Channel Depth}

The effect of the channel depth was studied using microchannels with $d=25 \mu \mathrm{m}$ and $40 \mu \mathrm{m}$, while maintaining the channel width $w=100 \mu \mathrm{m}$. The time evolution of the DSCG textures obtained for plasma-treated microchannels of depth $25 \mu \mathrm{m}$ and $40 \mu \mathrm{m}$ are shown in Figures A9 and A10; while Figures A11 and A12 capture the effect of the depth in homeotropic channels. On increasing the depth by $150 \%$ (i.e., from $10 \mu \mathrm{m}$ to $25 \mu \mathrm{m}$ deep), the M-phase appeared between 45 and $60 \mathrm{~min}$, whereas on increasing the depth by $300 \%$ (100 $\mu \mathrm{m}$ wide, from $10 \mu \mathrm{m}$ to $40 \mu \mathrm{m}$ deep), the M-phase appeared between 60 and $75 \mathrm{~min}$. Thus, the appearance of the M-phase, and hence, the nematic to M-phase transition, is retarded due to the increase in the microchannel depth. For the DMOAPtreated microchannels (homeotropic anchoring), the time evolution of the textures are shown in Figures A13 and A14, respectively. Here also, the transition time scale is stretched, relative to the control case $(d=10 \mu \mathrm{m})$ : the M-phase appears at about $60 \mathrm{~min}$ within the $100 \mu \mathrm{m}$ wide, $25 \mu \mathrm{m}$ deep channel; while no phase change was captured within the first $60 \mathrm{~min}$ within the $40 \mu \mathrm{m}$ deep channel. Thus, an increment in the channel depth counters the effects of the increment of the channel width: the two opposing effects can be effectively captured in terms of the channel aspect ratio $(A R=w / d)$. A careful choice of the aspect ratio should allow us to harness the trade-off between the transition timescales for tuning the nematic to M-phase transitions within microfluidic geometries.
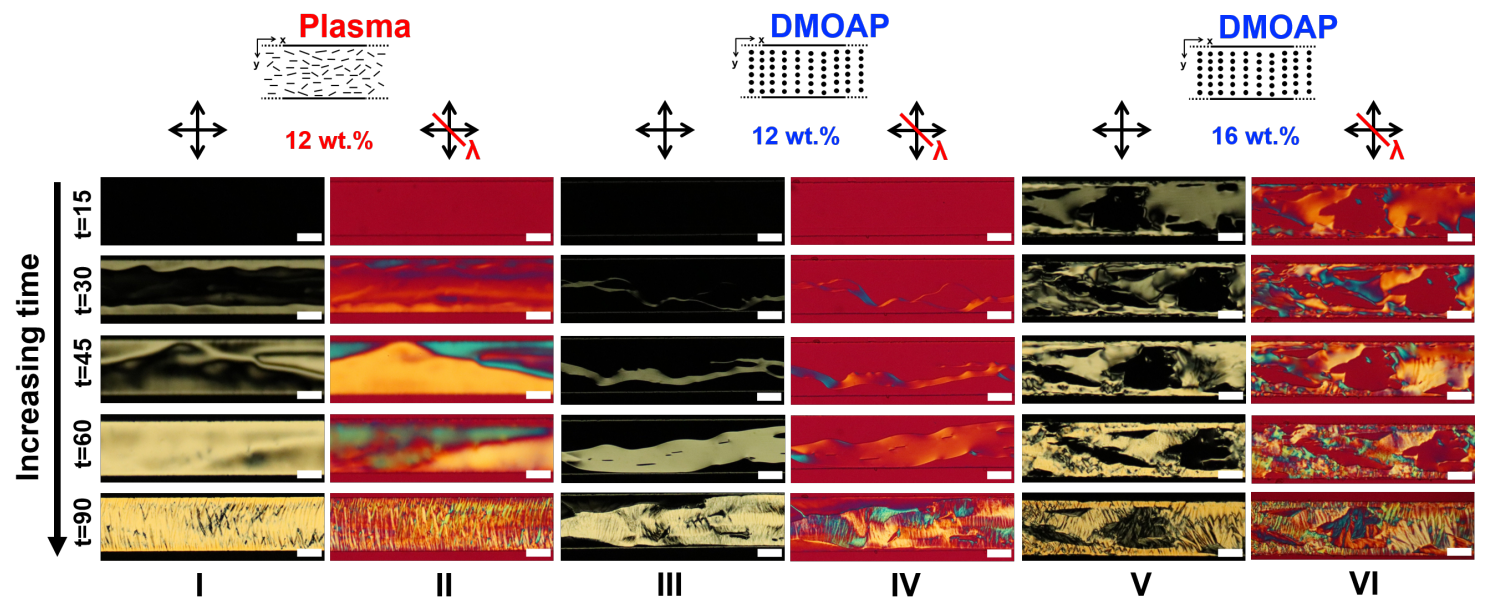

Figure 10. DSCG textures at $12 \mathrm{wt} . \%$ and $16 \mathrm{wt} . \%$, within a $200 \mu \mathrm{m}$ wide, $10 \mu \mathrm{m}$ deep microchannel. (I-IV) show textures exhibited by the nematic phase formed by $12 \mathrm{wt}$.\% DSCG, under degenerate planar and homeotropic anchoring conditions, respectively. While the planar channel transitioned from the isotropic to nematic to M-phase herringbone texture, the homeotropic channel transitioned from the isotropic to the spherulite texture over long times. (V,VI) present LCLC textures formed by 16 wt.\% DSCG, under homeotropic anchoring, observed through crossed polarizers and the $\lambda$-retardation plate. The sequence of transition was similar to that of the $12 \mathrm{wt} \%$ DSCG; however, the transitions were relatively faster due to the proximity of the $16 \mathrm{wt} . \%$ to the phase transition. Here, time (t) is in minutes after filling and sealing of the glass-PDMS microfluidic device. Scale bar: $200 \mu \mathrm{m}$.

Once LCLC textures stabilized within the channels, we observed qualitative differences in their appearance due to variations in the channel depths. The general features of respective textures are however detectable in both shallow and deeper channels, as shown in Figures A15 and A16 (for planar anchoring) and in Figure A17 (for homeotropic anchoring). Overall, the DSCG textures change from a uniform to a non-uniform appearance with the increase in the channel depth from $10 \mu \mathrm{m}$ to $40 \mu \mathrm{m}$, for both the degenerate 
(Figure A11) and the homeotropic (Figure A14) boundary conditions. Based on the above results, we concluded that the stability of the nematic and M-phase textures, and the transitions therein, depends on the channel dimensions and the surface anchoring conditions. Furthermore, the depth of the microchannel regulates the uniformity of the emergent textures: the lower the depth (i.e., stronger confinement), the more uniform is the stabilized texture, whereas within deeper channels, the textures become increasingly non-uniform, possibly due to increased bulk effects (due to relatively weak surface effects). Additionally, we used two other DSCG concentrations for our experiments: $12 \mathrm{wt} . \%$ (closer to the isotropic phase at the operating temperature) and $16 \mathrm{wt} . \%$ (closer to the M-phase) and studied the POM textures $200 \mu \mathrm{m}$ wide and $10 \mu \mathrm{m}$ microchannels. As shown in Figure 10 (I-IV), initially, no microfluidic texture was detected at $12 \mathrm{wt} . \%$ DSCG, under both degenerate planar and homeotropic boundary conditions. This is due to the presence of a high proportion of the isotropic phase in the DSCG solution. Then, between 45 and $60 \mathrm{~min}$, planar nematic textures emerged, which at about $90 \mathrm{~min}$ changed to the M-phase texture. The sequence of isotropic-nematic-M-phase transition confirms the gradual increase of the DSCG concentration within the microfluidic environment. At $16 \mathrm{wt} . \%$, within the DMOAP-treated microchannel (V-VI), a patchy homeotropic domain was observed (with regions of total light extinction), while the rest of the regions were birefringent, which ultimately transformed to the M-phase at around $60 \mathrm{~min}$.

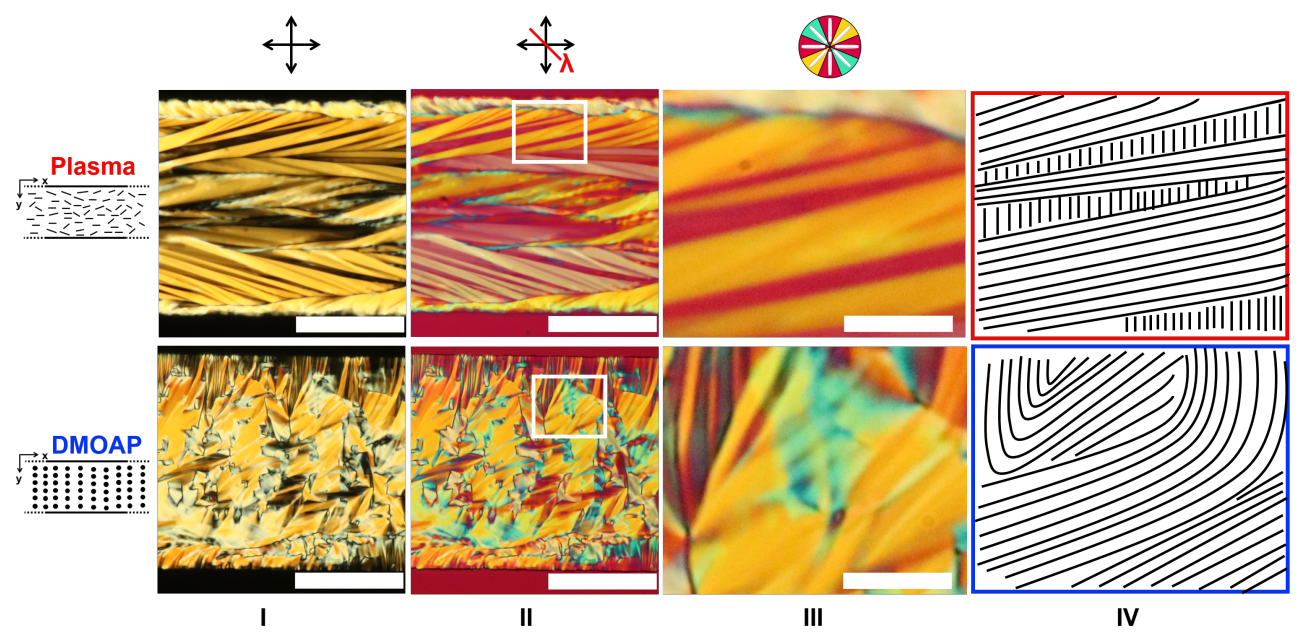

Figure 11. Comparative analysis of stable herringbone and spherulite microfluidic textures. By observing $14 \mathrm{wt}$.\% DSCG within the $200 \mu \mathrm{m}$ wide, $10 \mu \mathrm{m}$ deep channel, distinct anisotropic microscale textures are revealed for degenerate planar and homeotropic anchoring, respectively, here visualized between (I) crossed polarizers and (II) crossed polarizers with a $\lambda$-retardation plate. The polarized micrographs in (III) present zoomed-in regions from each of the samples, and (IV) presents the corresponding schematic of the integrated M-phase director field in 2D. Scale bar in (I,II): $100 \mu \mathrm{m}$; in (III): $20 \mu \mathrm{m}$.

\subsection{Comparing the Effects of Surface Treatment on the Stable LCLC Microfluidic Textures}

Figures 11 and A18 present a comparative analysis of the M-phase texture developed in PDMS-glass microchannels, under both plasma (degenerate planar) and DMOAP (homeotropic) surface treatments. For the microchannels with plasma treatment, the Mphase yields the herringbone texture, with an overall structural orientation along the channel length. For the DMOAP-treated microchannel, the M-phase elicits the spherulite texture, which aligns perpendicular to the channel walls, i.e., along the transverse direction (Figures 11 and A18). As indicated by the contact angle measurement of the $14 \mathrm{wt} . \%$ DSCG solution (Figure 1, Panels $f$ and g), plasma exposure renders both PDMS and glass surfaces hydrophilic, whereas the silane, DMOAP, makes the surface hydrophobic. Consequently, the nucleation, growth, and propagation of the textures will be locally influenced by the nature of the boundary conditions. In the planar case, the discotic stacks in the nematic or 
M-phase are expected to align along the planar domains imposed by the plasma treatment. This, with the additional constraint from the long axis of the microchannel, guides the herringbone structure along the channel length. For the homeotropic case, the spherulite texture emerges due to the normal alignment of the discotics on the channel surfaces and is thus constrained to grow from the ceiling to the floor and from wall-to-wall of the microchannel. Thus, we do not see any structural bias along the channel length.

\subsection{Discussions}

In order to gain qualitative insights into the textural transitions, we looked into the impact of air plasma and DMOAP treatments on the PDMS surface morphology. It has been known that plasma treatment of the PDMS surface causes nano-structuring of PDMS and increases the water contact angles depending on the duration and intensity of the plasma treatment used [60-63]. Bodas et al. showed the notable cracks (through SEM) of the PDMS upon $150 \mathrm{~W}, 15 \mathrm{~min}$, plasma treatment [60]. On the other hand, Hillborg et al. employed $40 \mathrm{~W}, 3 \mathrm{~min}$, plasma treatment and observed insignificant surface morphological changes (through SEM and AFM) [63]. From the surface hydro-philicity/-phobicity angle (a surface parameter modified reproducibly by air plasma exposure of glass and PDMS), Ruben et al. reported enhanced capillary flow within PDMS microchannels that were hydrophilic [64]. Complementarily, from a membrane perspective, hydrophobic species, depending on the molecular size, can permeate readily through PDMS $[65,66]$. Since DSCG is a bulkier molecule with a much higher solubility value $(195.3 \mathrm{mg} / \mathrm{mL}$ [67]) compared to small molecules like quinine or Nile red, we could rule out the possibility of DSCG permeation in PDMS. On the other hand, hydrophilized PDMS surfaces (after plasma exposure) would enhance the permeation of water molecules through PDMS; hydrophobic PDMS (native or DMOAP-treated) will hinder the permeation of water molecules. Consequently, depending on the surface treatment used in our experiments (plasma vs. DMOAP), we expect water uptake by the PDMS surfaces to vary. Consequently, this impacts the timescales over which the water molecules disappear from the DSCG solution, triggering an increase in the local DSCG concentration, ultimately pushing the solution closer to the nematic to M-phase transition. Once the M-phase nucleates locally, it propagates through the channel thereafter as more water molecules permeate into the PDMS over time. However, the rate at which the water molecules enter PDMS is determined by the surface hydro-philic/-phobic characteristics. The experimental timescales of the nematic-M-phase transition in our study confirm this and that the surface properties along with geometric dimensions mediate the textural dynamics of LCLC solutions under static conditions.

It is evident from the time dependent optical observations that the nematic phase of 14 wt.\% DSCG, within both plasma- and DMOAP-treated microchannels, exhibited a phase transition from the nematic to the more ordered columnar M-phase. It is known that the M-phase occurs at a higher concentration of DSCG, and for a nematic-M-phase transition to occur from a nematic phase, there has to be a change in the concentration of DSCG (unless the temperature of the environment changes, which in our experiments was kept fixed). The DSCG concentration can change either by the variation of the temperature or due to the loss of water from the solution. As all measurements were done at a constant temperature, in evaporation-proof microfluidic devices, the change in the initial concentration of the DSCG solution has to come from the loss of water due to permeation into PDMS. To confirm that the nematic to M-phase transition results due to the permeation of water molecules into the PDMS, we compared the stability of the DSCG textures (for the $14 \mathrm{wt} . \%$ DSCG solution) within glass capillaries, for both native and DMOAP-treated samples. As shown in Figures A19 and A20, for untreated glass capillaries (weakly homeotropic) and DMOAP-treated glass capillaries (strong homeotropic anchoring), respectively, the textures for degenerate planar and homeotropic alignment were observed. The time dependent texture analysis did not reveal the nematic to M-phase transition, regardless of the surface treatment, thus confirming the role of PDMS surfaces in taking up water molecules. Furthermore, we studied the nematic phase of the $14 \mathrm{wt} . \%$ DSCG solution 
by keeping one end of the glass capillary open to allow the evaporation of water. The time evolution of texture showed an increase in the birefringence, indicating a transition to a more ordered phase, though no herringbone M-phase texture was observed due to the weakly homeotropic conditions within the native capillaries (Figure A21). The above combination of control tests confirms that the M-phase development within the PDMSglass microchannels is mediated by the surface attributes of PDMS, which is currently being investigated further.

We studied the $12 \mathrm{wt} . \%$ DSCG solution in a plasma-treated PDMS-glass microchannel Figure A22. As this concentration is close to the isotropic-nematic phase transition temperature (see the phase diagram in Figure 1), one should expect that the system has to first reach a concentration of DSCG that shows a stable nematic phase, and then, the N-M-phase transition follows. We filled a plasma-treated PDMS-glass microchannel with the $12 \mathrm{wt} . \%$ solution and sealed it, then as discussed previously, we carried out the POM time lapse imaging (imaged every $30 \mathrm{~s}$ ). As hypothesized, initially, the system was observed to be in the isotropic phase, with the nematic phase emerging after about $25 \mathrm{~min}$. Thereafter, the system stayed in the nematic phase for about $255 \mathrm{~min}$, before the M-phase finally appeared. This confirmatory test consolidates our conclusions that the surface properties of PDMS induces systematic changes in the initial concentration of DSCG, which ultimately lead to the formation of the spontaneous, yet tunable, LCLC phase transitions. A detailed quantitative analysis of the surface-mediated phase transition will be discussed elsewhere [55].

\section{Conclusions}

We exploit a simple and flexible soft lithography technique to create PDMS-glass microfluidic devices for studying the nucleation, growth, and stability of LCLC textures. The temporal study carried out here reveals the spontaneous (and unexpected) emergence of M-phase textures, with DSCG solutions that are originally in the nematic phase. Our comprehensive polarization optical study demonstrates that the static LCLC microfluidic textures, within standard PDMS-glass microfluidic devices, are far from trivial due to their dependence on the following key parameters:

(i) microchannel dimensions (confinement effect),

(ii) surface anchoring (anchoring effects, specifically in relation to the PDMS surface), and

(iii) experimental timescale (spontaneous surface-mediated effects).

The three fundamental variables determine the local and temporal no-flow LCLC microfluidic textures, giving us a set of key experimental parameters which could be potentially employed to register the initial conditions for microfluidic flow experiments.

Table 1 summarizes the textural manifestations of DSCG solutions in PDMS-glass microchannels and the timescales over which various surface-induced phase transitions occur. Multiple experimental replicates confirm the reproducibility of the values included in Table 1 , for a range of aspect ratios $(w / d)$ of channels possessing either degenerate planar or homeotropic boundary conditions (Figure 9). Although plasma treatment of microchannels successfully imposed degenerate planar conditions (for both the thermotropic and LCLC nematic phase), the DMOAP treatment, used frequently to generate homeotropic alignment, failed to have a similar effect on DSCG solutions at 12 and $14 \mathrm{wt} . \%$ concentrations. However, at $16 \mathrm{wt} . \%$, we observed larger homeotropic patches (leading to a higher cumulative area showing homeotropic texture), with longer temporal stability. We conclude that silane derivatives (e.g., DMOAP), usually employed for generating homeotropic alignment for thermotropic LCs, may not be as effective for inducing normal surface anchoring of the LCLCs within PDMS-glass devices. Based on a previous report by Tone et al. both hydrophobicity and chemical affinity could play a role in inducing homeotropic alignment. Here, materials with low surface energy — without alkyl chains-could be considered as suitable alternatives to generate homeotropic alignment, for instance polybutadiene and styrene-butadiene-styrene [24]. These materials can work for glass devices; however, there are a few technical hurdles in coating PDMS-glass microchannels with these materials, 
primarily due to the dissolution step (with organic solvents), which can cause swelling of the PDMS, thereby modifying the microfluidic geometry.

The key and the most striking result of this work as summarized in Figures 9 and A1 is the temporal variation of lyotropic textures induced by a spontaneous phase transition from the nematic to the M-phase. The time of first appearance of the M-phase varied inversely with the channel aspect ratio $(A R=w / d)$. This trend was most pronounced in the $14 \mathrm{wt} . \%$ sample. By changing the channel dimensions, surface anchoring, and the concentration of the LCLC material, we could systematically tune both the nature of the characteristic texture and the temporal dynamics of the nematic to M-phase transition. Additionally, the texture of the M-phase depended on the surface anchoring conditions: herringbone texture under degenerate planar conditions; and spherulite texture under homeotropic conditions. Furthermore, both herringbone and spherulite textures manifested global orientations: while the herringbone texture was oriented along the channel length, the spherulite texture was oriented perpendicular to the channel length (in the transverse direction). The temporal dynamics of the nematic to M-phase transition and the final M-phase texture (stable over a long time) could be controlled by varying the aspect ratio or, independently, the channel width and the depth. Our straightforward strategy to employ LCLC self-organization within a soft-lithography-based microfluidic platform considerably widens the scope of LCLCs, for both applied and fundamental research, and sets the stage for future studies on the flow behavior LCLCs in strict microfluidic environments. Interestingly, even the static no-flow textures hold promising prospects for potential applications in LCLC-mediated transport processes, for instance, in regulating the anisotropic diffusion coefficients locally over time.

Table 1. Effect of the channel dimension (variation in width or depth shown as underlined), surface anchoring (degenerate planar vs. homeotropic), and DSCG concentration (12,14 and $16 \mathrm{wt} . \%)$ on the nematic to M-phase transition time.

\begin{tabular}{|c|c|c|c|}
\hline $\begin{array}{l}\text { Channel Dimension } \\
\qquad \mathbf{w} \times \mathrm{d}(\mu \mathrm{m})\end{array}$ & Anchoring Condition & $\begin{array}{c}\text { DSCG Concentration } \\
(\%)\end{array}$ & $\begin{array}{c}\text { Nematic to M-Phase } \\
\text { Transition } \\
\text { Time (min) }\end{array}$ \\
\hline $100 \times 10$ & Degenerate planar & 14 & 15 \\
\hline$\overline{100} \times 10$ & Homeotropic & 14 & 15 \\
\hline$\underline{200} \times 10$ & Degenerate planar & 14 & $30-45$ \\
\hline$\overline{200} \times 10$ & Homeotropic & 14 & $30-45$ \\
\hline$\underline{345} \times 10$ & Degenerate planar & 14 & 45 \\
\hline$\overline{345} \times 10$ & Homeotropic & 14 & $30-45$ \\
\hline $100 \times \underline{25}$ & Degenerate planar & 14 & $45-60$ \\
\hline $100 \times \overline{25}$ & Homeotropic & 14 & 60 \\
\hline $100 \times \underline{40}$ & Degenerate planar & 14 & $60-75$ \\
\hline $100 \times \overline{40}$ & Homeotropic & 14 & 75 \\
\hline $200 \times \underline{15}$ & Degenerate planar & 14 & 45 \\
\hline $200 \times 15$ & Homeotropic & 14 & $45-60$ \\
\hline $200 \times 10$ & Degenerate planar & 12 & 90 \\
\hline $200 \times 10$ & Homeotropic & 12 & 90 \\
\hline $200 \times 10$ & Homeotropic & 16 & 60 \\
\hline
\end{tabular}

As shown through the time lapse image sequence in Figure 12, defect lines near a microscale inclusion (the channel and inclusion were DMOAP-treated) can transition over time-purely due to surface-mediated effects-thereby triggering microscale transport, even in absence of external pressure gradients [55]. Investigations on the dynamics of LCLCs under flow within PDMS-glass microfluidic devices is currently underway and can be extended to other systems to study surface-mediated phase transitions, for instance in water-based lyotropic liquid crystals systems such as peptides, liquid crystal polymers, and viruses. The temporal stability of textures can result in significant real-world applications, in particular to capture the dynamics of in biological systems, where transport processes 
can respond to (or be modulated by) the compliance, surface, and morphological attributes of the system.
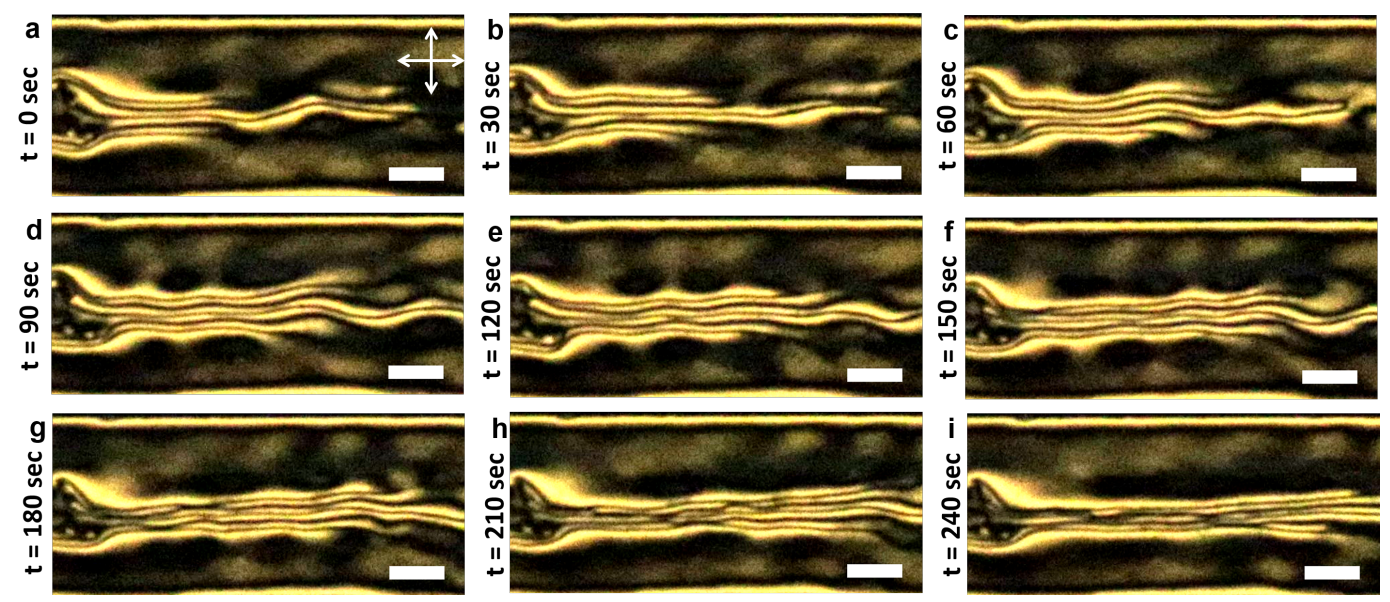

Figure 12. Pseudo flow textures exhibited by the nematic phase of $14 \mathrm{wt}$.\% DSCG between crossed polarizers, imaged within a $345 \mu \mathrm{m}$ wide, $10 \mu \mathrm{m}$ deep microchannel with homeotropic anchoring. (a-i) Time evolution of the disclination lines. Scale bar: $100 \mu \mathrm{m}$.

Author Contributions: A.S. (Anshul Sharma) and A.S. (Anupam Sengupta) designed the research, performed the experiments, and analyzed data. All authors interpreted the data and wrote the paper. All authors have read and agreed to the published version of the manuscript.

Funding: This work was supported by the ATTRACT Investigator Grant to A.S.G. (Grant Number: A17/MS/ 11572821/MBRACE) of the Luxembourg National Research Fund.

Institutional Review Board Statement: Not applicable.

Informed Consent Statement: Not applicable.

Data Availability Statement: For all supporting data, contact the corresponding author or refer to the Appendix A.

Acknowledgments: The authors thank the Luxembourg National Research Fund for supporting this work through the ATTRACT Investigator Grant (to A.S., Grant Number: A17/MS/ 11572821/MBRACE), and are grateful to M.G. Mazza, A. Ghoshal, and J. Dhar for valuable discussions during the course of this work.

Conflicts of Interest: The authors declare no conflict of interest. 


\section{Appendix A. Supplementary Figures}

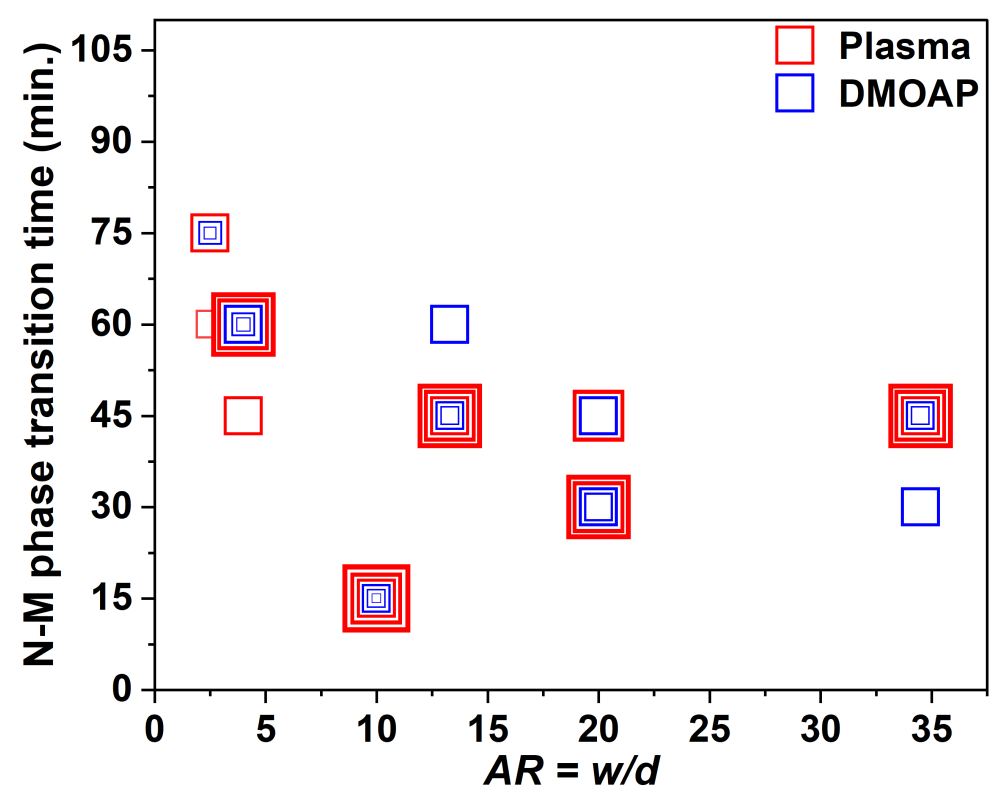

Figure A1. Dependence of the nematic to M-phase transition time on the channel aspect ratio, shown here for the experimental replicates covered in this study (34 replicates for $14 \mathrm{wt}$ \% DSCG concentration, under both plasma and DMOAP surface treatments). Red squares indicate degenerate planar data, while blue squares represent homeotropic data. For $\mathrm{t}=15 \mathrm{~min}$ and $A R=10$, the plot shows 3 overlying data points for plasma (red square), and 3 overlying data points for DMOAP (blue square); for $\mathrm{t}=30 \mathrm{~min}$ and $A R=20,2$ overlying data points are shown for plasma (red square), and 2 overlying data points for DMOAP (blue square); for $\mathrm{t}=45 \mathrm{~min}$ and $A R=13.3$, the plot shows 3 overlying data points for plasma (red square), and 2 overlying data points for DMOAP (blue square); for $\mathrm{t}=45 \mathrm{~min}$ and $A R=20$, the plot shows 1 overlying data points for plasma (red square), and 1 overlying data point for DMOAP (blue square); for $\mathrm{t}=45 \mathrm{~min}$ and $A R=34.5$, the plot shows 3 overlying data points for plasma (red square), and 2 overlying data points for DMOAP (blue square); for $\mathrm{t}=60 \mathrm{~min}$ and $A R=2.5$, the plot shows single data point for plasma (red square); and $A R=4$, the plot shows 2 overlying data points for plasma (red square), and 2 overlying data points for DMOAP (blue square); and for $\mathrm{t}=75 \mathrm{~min}$ and $A R=2.5$, the plot shows 2 overlying data points for plasma (red square), and 2 overlying data points for DMOAP (blue square).

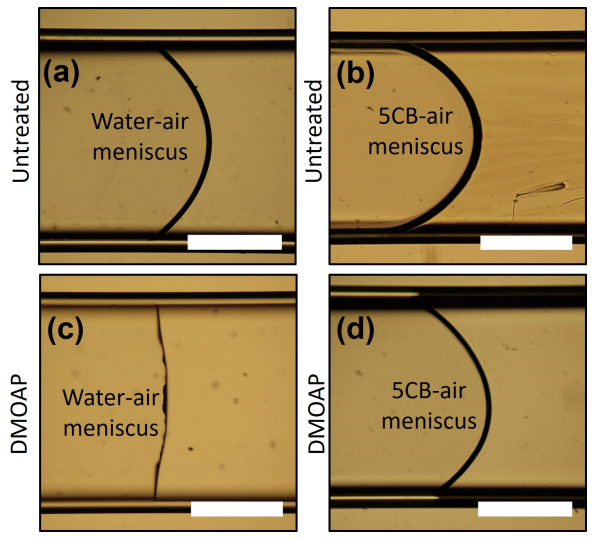

Figure A2. Optical micrographs of menisci within glass capillaries (viewed horizontally under microscope) with dimensions $100 \mu \mathrm{m} \times 1000 \mu \mathrm{m}$ (cross-section dimensions), without any treatment: (a) Water; (b) 5CB (filled in nematic phase). Optical micrograph of glass capillary after DMOAP treatment: (c) water and (d) 5CB (filled in isotropic phase). Scale bar: $500 \mu \mathrm{m}$. 


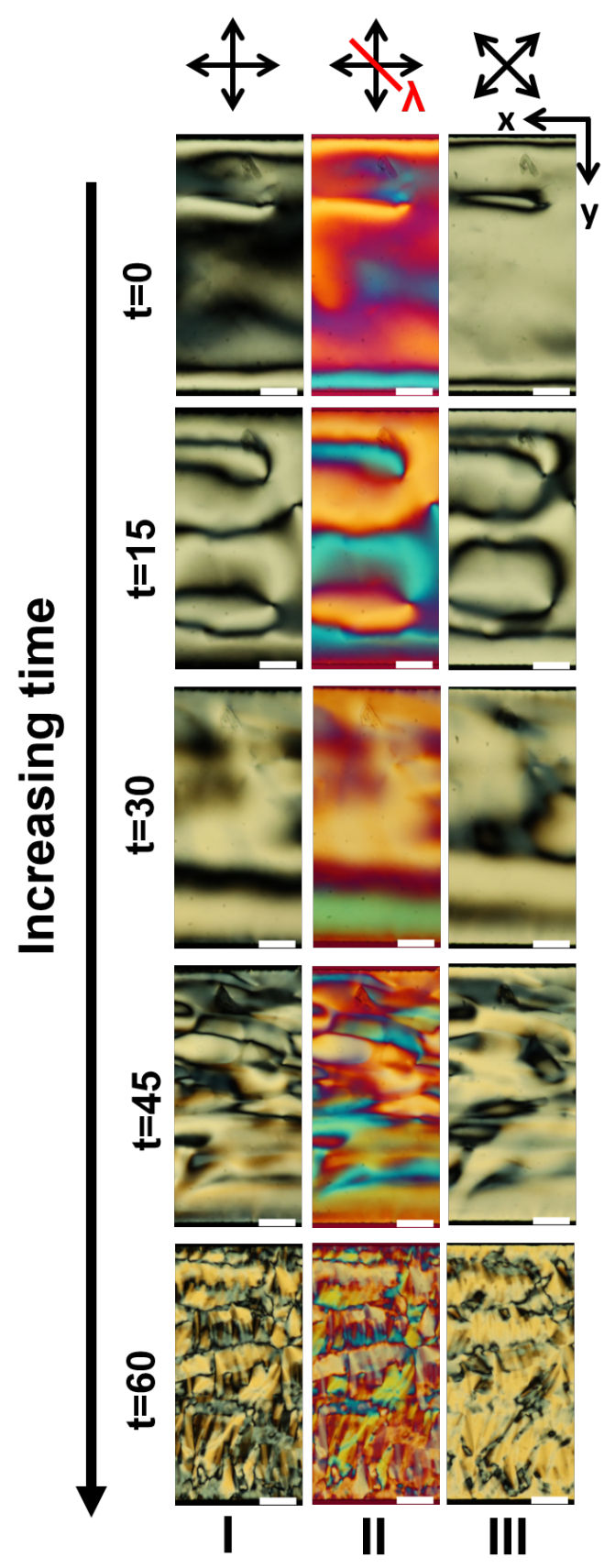

Figure A3. Degenerate planar anchoring within wider channels, here using $345 \mu \mathrm{m}$ wide, $10 \mu \mathrm{m}$ deep microfluidic device. The transition to herringbone texture is accelerated in wider channels, as captured by the time lapse imaging with polarizers at various configurations:(I) between crossed polarizers; (II) crossed polarizers with the $\lambda$-plate; (III) crossed polarizers with channel at $45^{\circ}$ relative to the polarizer. Here, time $(t)$ is in minutes after the cooling ramp is completed. Scale bar: $50 \mu \mathrm{m}$. 


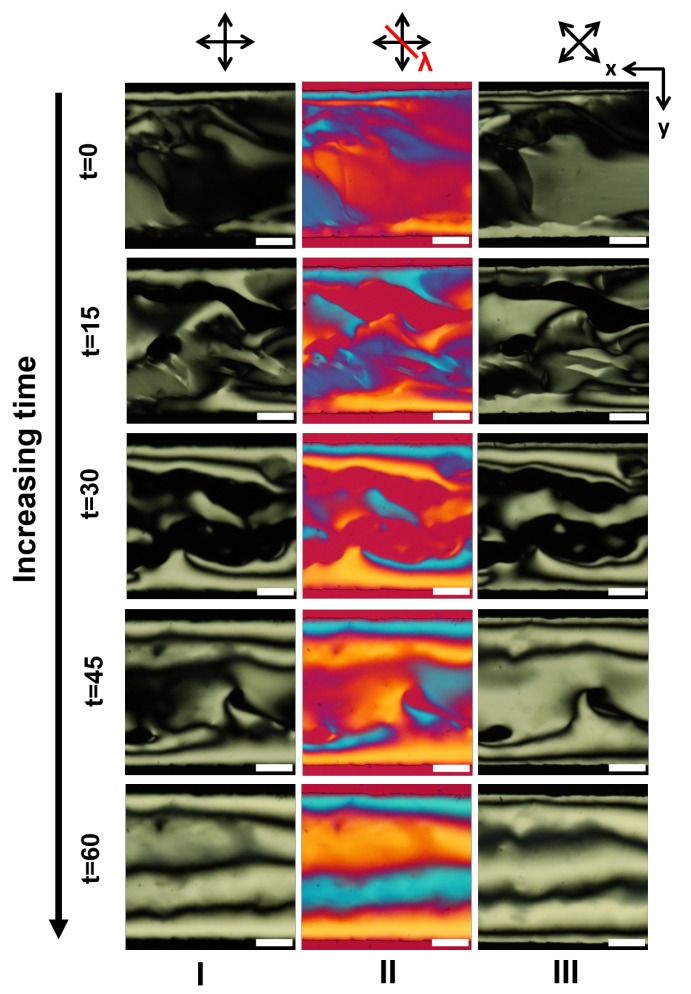

Figure A4. LCLC microfluidic textures in $200 \mu \mathrm{m}$ wide, $10 \mu \mathrm{m}$ deep microchannels treated for homeotropic anchoring. The textural evolution was observed over time, using polarizing optical microscopy. The configuration of the polarizer and $\lambda$-retardation plate are presented at the top of each image. Here, time (t) is in minutes after cooling ramp is concluded. (I-III) shows the POM micrographs obtained from a combination of different polarizer orientations at the corresponding time. Scale bar: $50 \mu \mathrm{m}$.

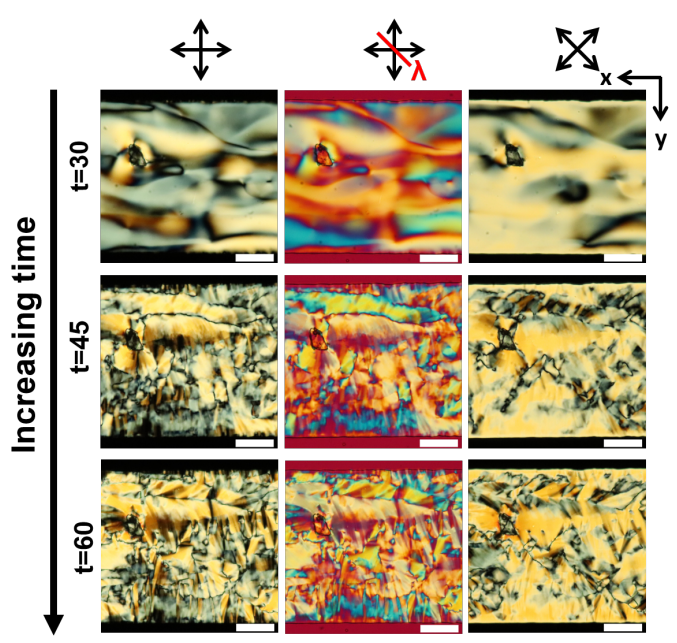

Figure A5. Long-term textures in $200 \mu \mathrm{m}$ wide, $10 \mu \mathrm{m}$ deep microchannels treated for homeotropic anchoring. The nucleation and propagation of M-phase can be observed here, imaged between polarizers under different configurations as described above. Here, time $(t)$ is in minutes after cooling ramp is concluded. Scale bar: $50 \mu \mathrm{m}$. 


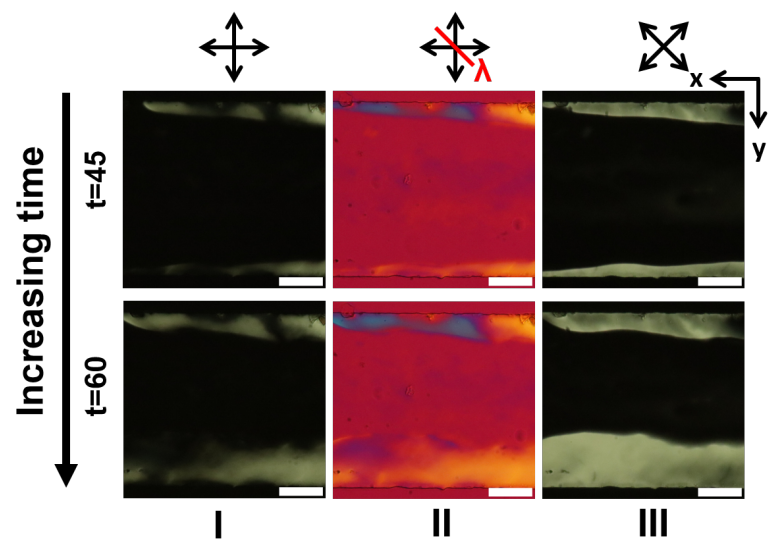

Figure A6. Patchy homeotropic anchoring in certain regions within the DMOAP treated $200 \mu \mathrm{m}$ wide, $10 \mu \mathrm{m}$ deep microchannel, with 14 wt.\% DSCG solution. The textures were stable over long timescales, observed here between polarizers under different configurations, as described above. Here, time ( $t$ ) is in minutes after cooling ramp is concluded. (I-III) shows the POM micrographs obtained from a combination of different polarizer orientations at the corresponding time. Scale bar: $50 \mu \mathrm{m}$.

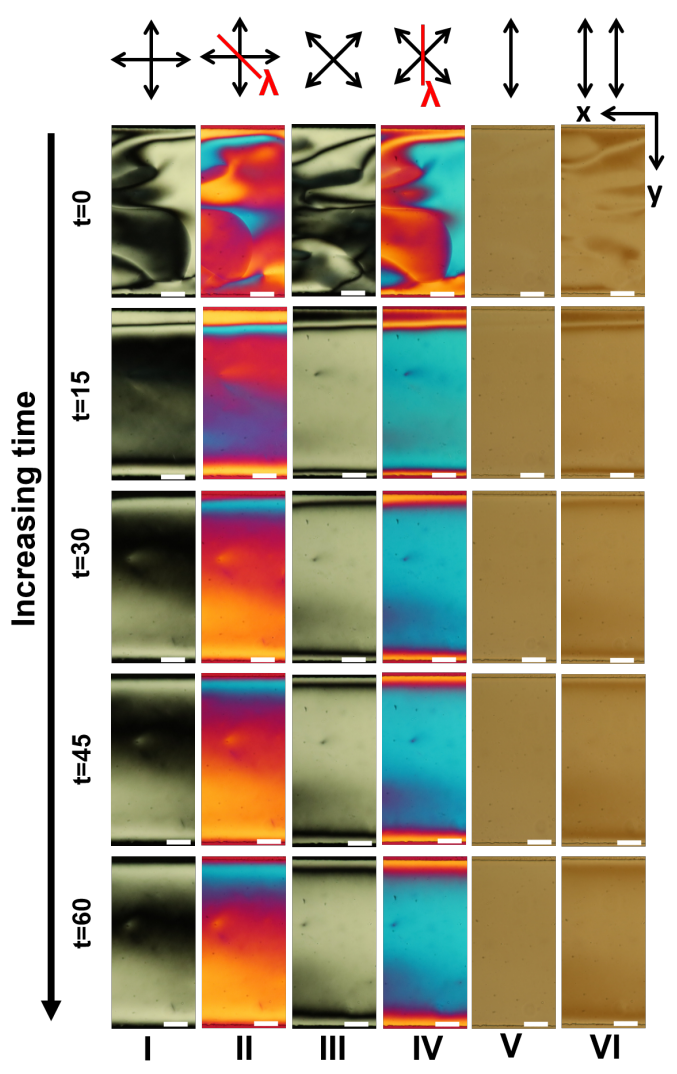

Figure A7. LCLC textures within wider channels possessing homeotropic anchoring, here within $345 \mu \mathrm{m}$ wide, $10 \mu \mathrm{m}$ deep microchannel, leading to the pseudo planar texture (14 wt.\% DSCG). Overall, the texture resembled that of planar or tilted orientation. Imaging details are noted at the top of each image. Here, time $(t)$ is in minutes after cooling ramp is concluded. (I-VI) shows the POM micrographs obtained from a combination of different polarizer orientations at the corresponding time. Scale bar: $50 \mu \mathrm{m}$. 


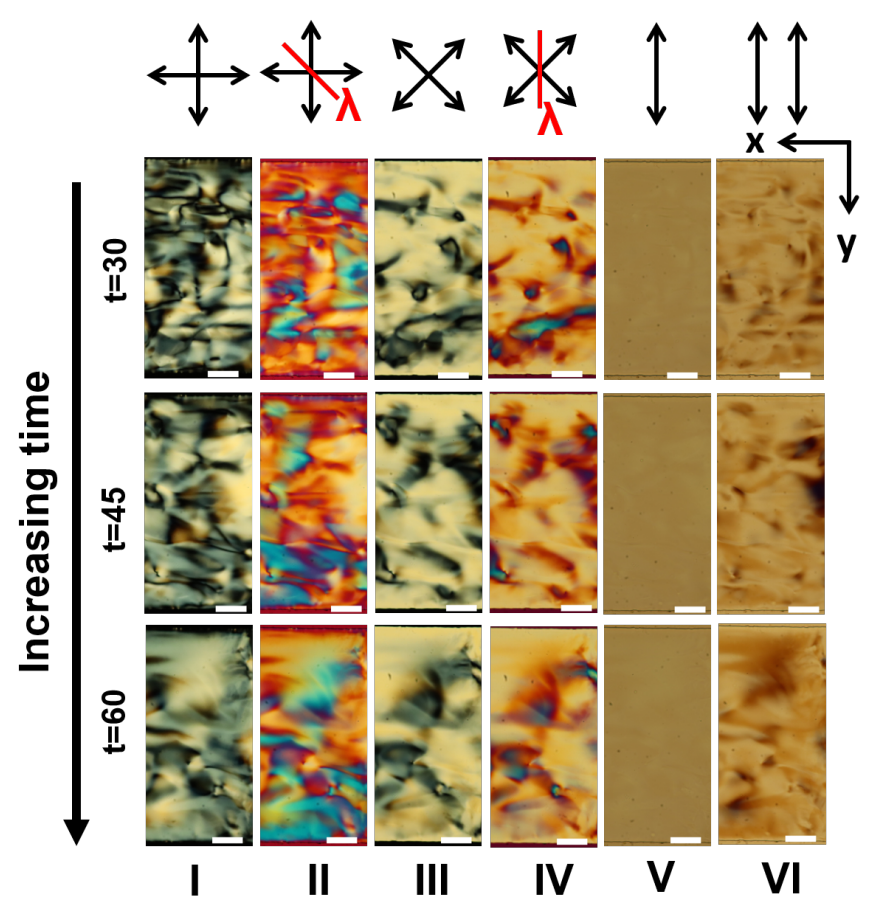

Figure A8. Gradual formation of the spherulite texture within DMOAP treated microchannel (345 $\mu \mathrm{m}$ wide, $10 \mu \mathrm{m}$ deep), with $14 \mathrm{wt}$ \% DSCG. Imaging started at $30 \mathrm{~min}$ after the end of the cooling ramp, and lasted till $60 \mathrm{~min}$ : (I) between crossed polarizers; (II) crossed polarizers and $\lambda$-plate; (III) crossed polarizers at $45^{\circ}$ relative to channel length; (IV) under crossed polarizers with $45^{\circ}$ rotation and $\lambda$-plate; (V) with only analyzer and (VI) analyzer parallel to the polarizer, with both perpendicular to the channel length. Here, time $(t)$ is in minutes after cooling ramp is concluded. Scale bar: $50 \mu \mathrm{m}$.

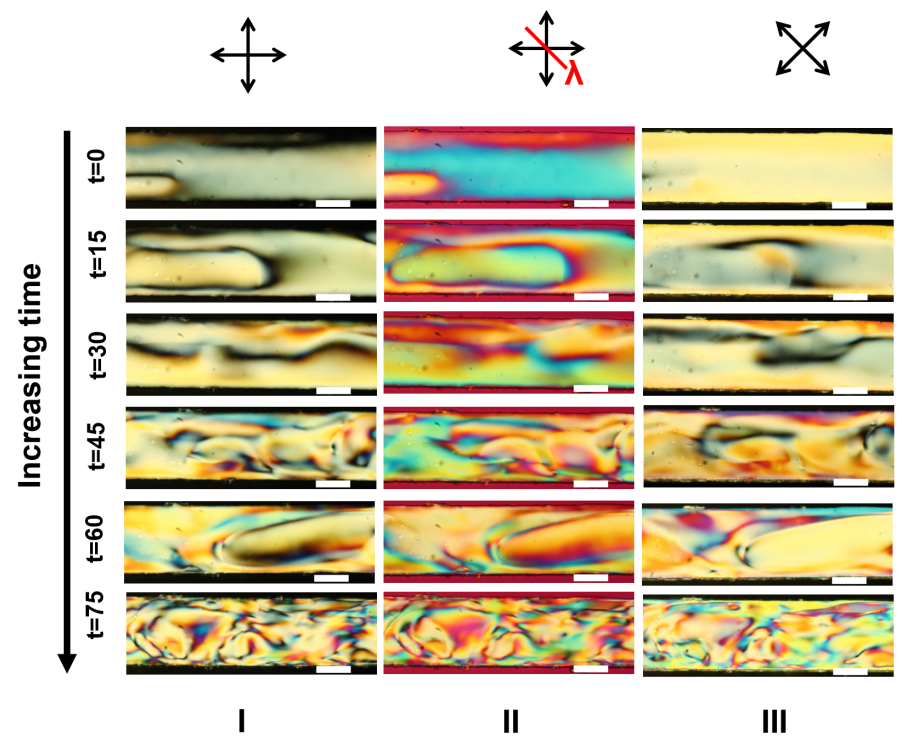

Figure A9. Degenerate planar anchoring-(100 $\mu \mathrm{m}$ wide, $25 \mu \mathrm{m}$ deep)-14 wt.\% DSCG. Evolution of textures exhibited as a function of time under various configurations of the polarizers. Polarizing optical micrographs show the top view of the channel from $t=0$ to $75 \mathrm{~min}$, images were taken as per the scheme discussed in the previous images. Here, time $(t)$ is in minutes after ramp is completed. (I-III) shows the POM micrographs obtained from a combination of different polarizer orientations at the corresponding time. Scale bar: $50 \mu \mathrm{m}$. 


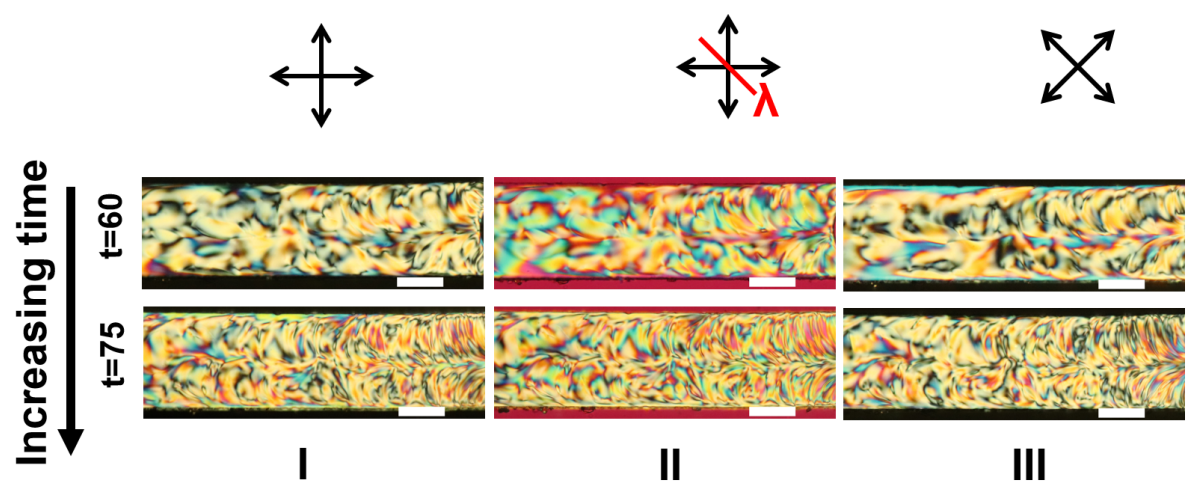

Figure A10. M-phase texture in $100 \mu \mathrm{m}$ wide, $25 \mu \mathrm{m}$ deep microchannel. Evolution of textures exhibited as a function of time under various conditions. Polarizing optical micrographs showing the top view of the channel from $\mathrm{t}=60 \mathrm{to} 75 \mathrm{~min}$. Evolution of textures under crossed polarizers; images were taken as per the scheme discussed in the previous images. Here, time ( $t$ ) is in minutes after ramp is completed. (I-III) shows the POM micrographs obtained from a combination of different polarizer orientations at the corresponding time. Scale bar: $50 \mu \mathrm{m}$.

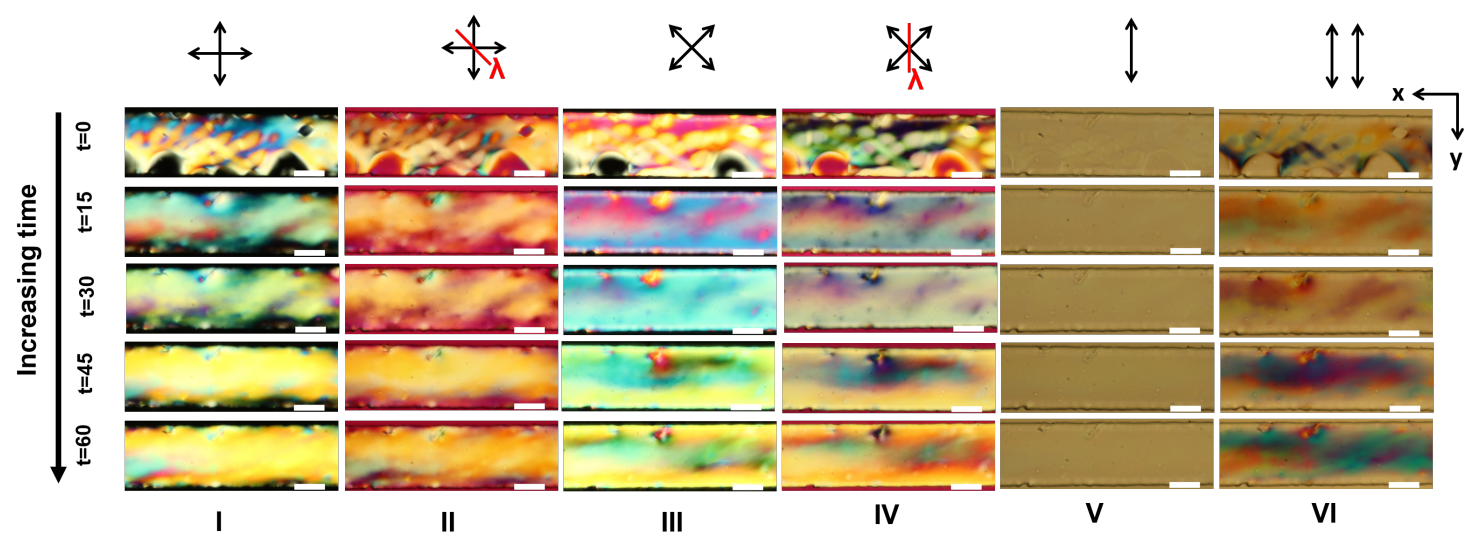

Figure A11. Degenerate planar anchoring textures in $100 \mu \mathrm{m}$ wide, $40 \mu \mathrm{m}$ deep channel. Evolution of textures as a function of time under various polarizer configurations, as described in previous images. Here, time ( $t$ ) is in minutes after cooling ramp is concluded. (I-VI) shows the POM micrographs obtained from a combination of different polarizer orientations at the corresponding time. Scale bar: $50 \mu \mathrm{m}$.

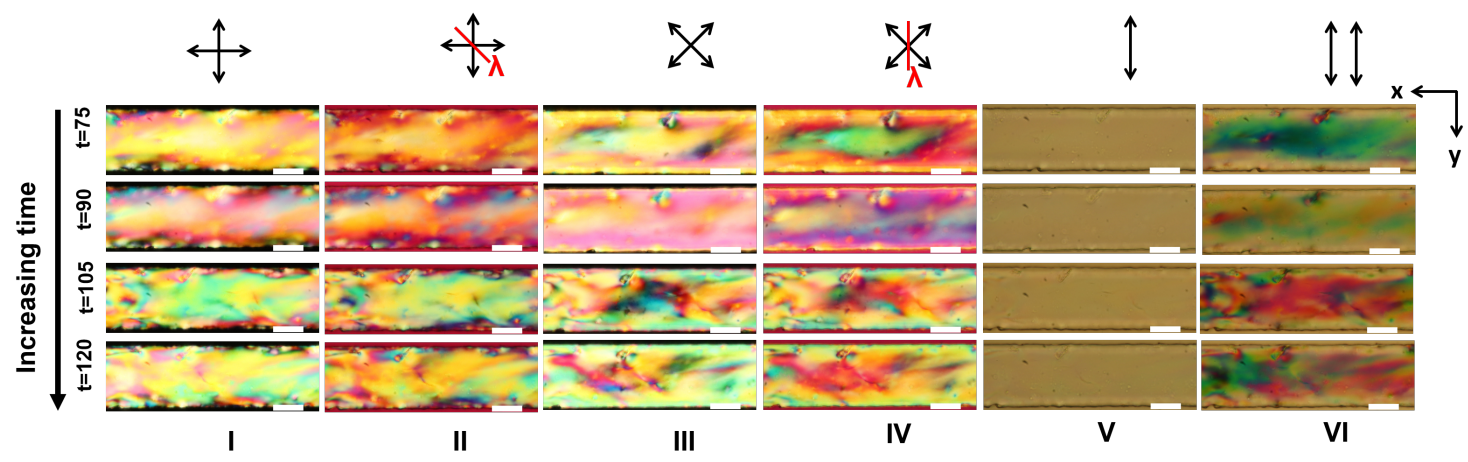

Figure A12. Long-term textures in $100 \mu \mathrm{m}$ wide, $40 \mu \mathrm{m}$ deep channels. The absence of the well-aligned herringbone texture, under degenerate planar boundary conditions, suggests that the textural transitions are hindered within deeper channels. Here, time ( $t$ ) is in minutes after cooling ramp is concluded. (I-VI) shows the POM micrographs obtained from a combination of different polarizer orientations at the corresponding time. Scale bar: $50 \mu \mathrm{m}$. 


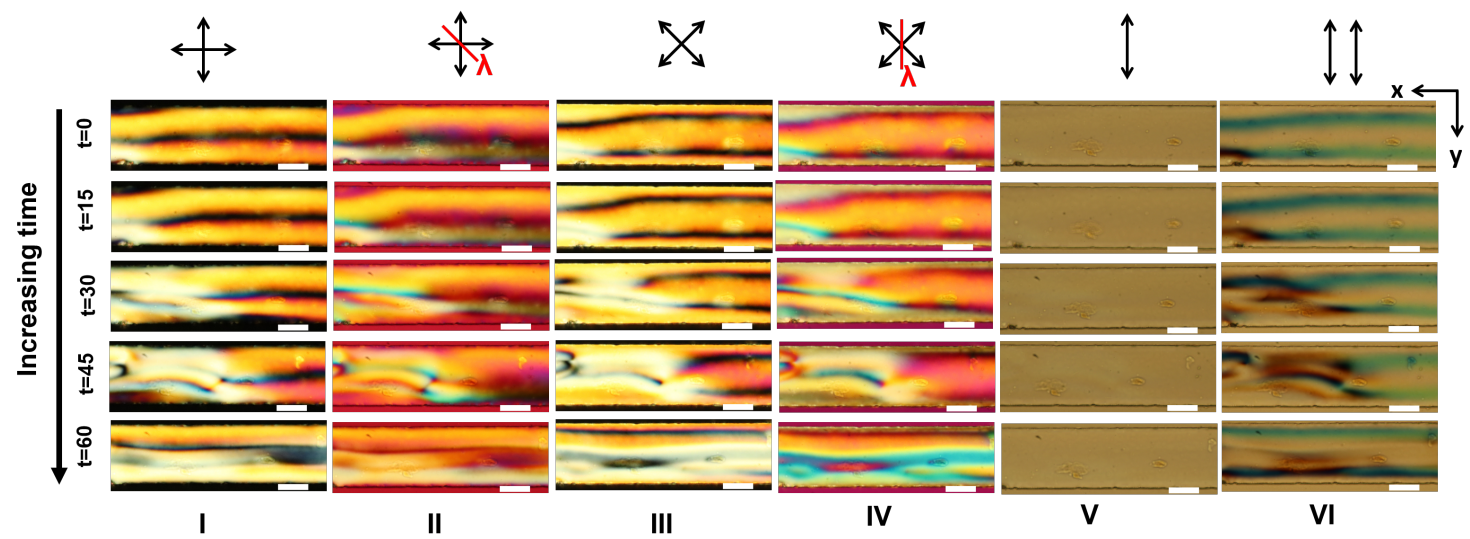

Figure A13. Effect of the channel depth on the DSCG texture, here observed within $100 \mu \mathrm{m}$ wide, $25 \mu \mathrm{m}$ deep microchannel possessing homeotropic anchoring conditions. The concentration of DSCG was $14 \mathrm{wt}$ \%. Polarizing optical micrographs were taken using same configuration of polarizers and $\lambda$-plate, as described previously. Here, time (t) is in minutes after cooling ramp is concluded. (I-VI) shows the POM micrographs obtained from a combination of different polarizer orientations at the corresponding time. Scale bar: $50 \mu \mathrm{m}$.

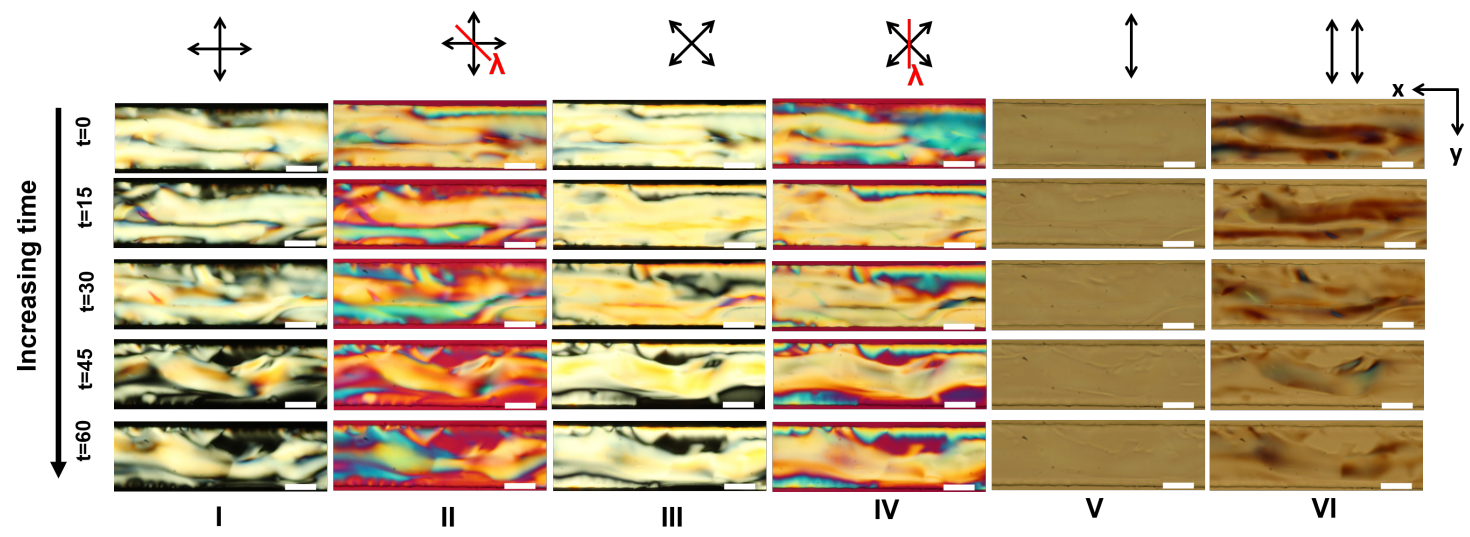

Figure A14. Loss of textural uniformity upon increasing the channel depth, here observed within $100 \mu \mathrm{m}$ wide, $40 \mu \mathrm{m}$ deep microchannel, for the $14 \mathrm{wt}$.\% DSCG. Time ( $\mathrm{t}$ ) is in minutes after cooling ramp is concluded. (I-VI) shows the POM micrographs obtained from a combination of different polarizer orientations at the corresponding time. Scale bar: $50 \mu \mathrm{m}$. 


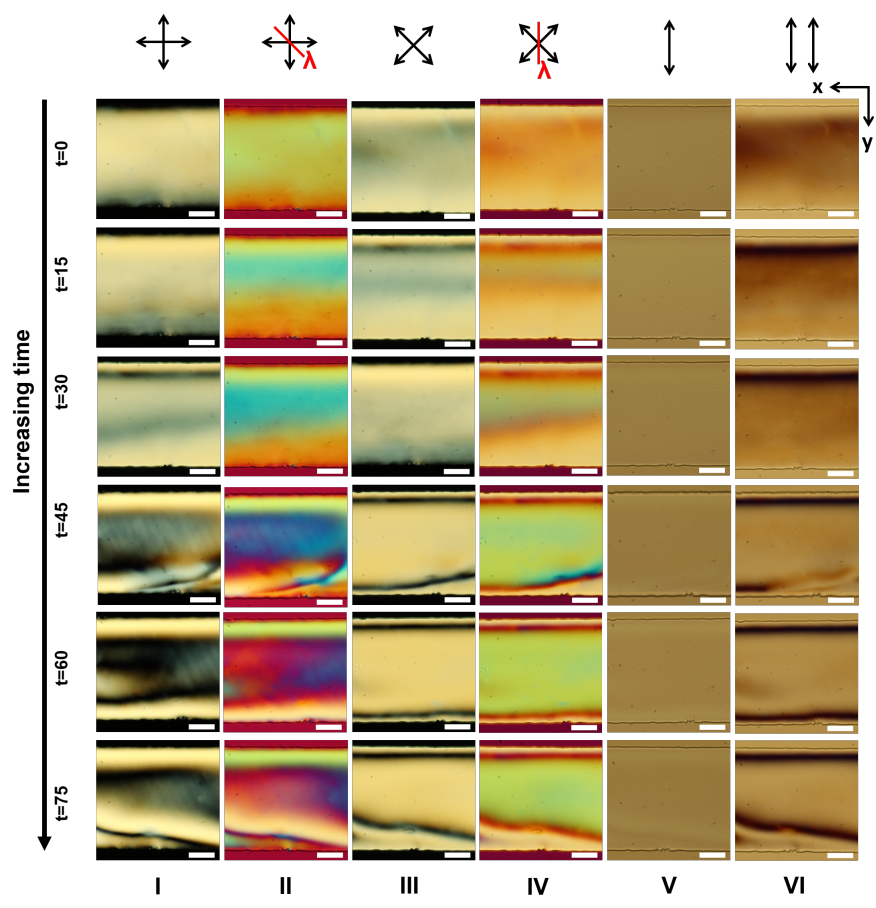

Figure A15. DSCG textures within $200 \mu \mathrm{m}$ wide, $15 \mu \mathrm{m}$ deep microchannel possessing degenerate planar anchoring, here observed with 14 wt.\% DSCG. Here, time (t) is in minutes after cooling ramp is concluded. (I-VI) shows the POM micrographs obtained from a combination of different polarizer orientations at the corresponding time. Scale bar: $50 \mu \mathrm{m}$.

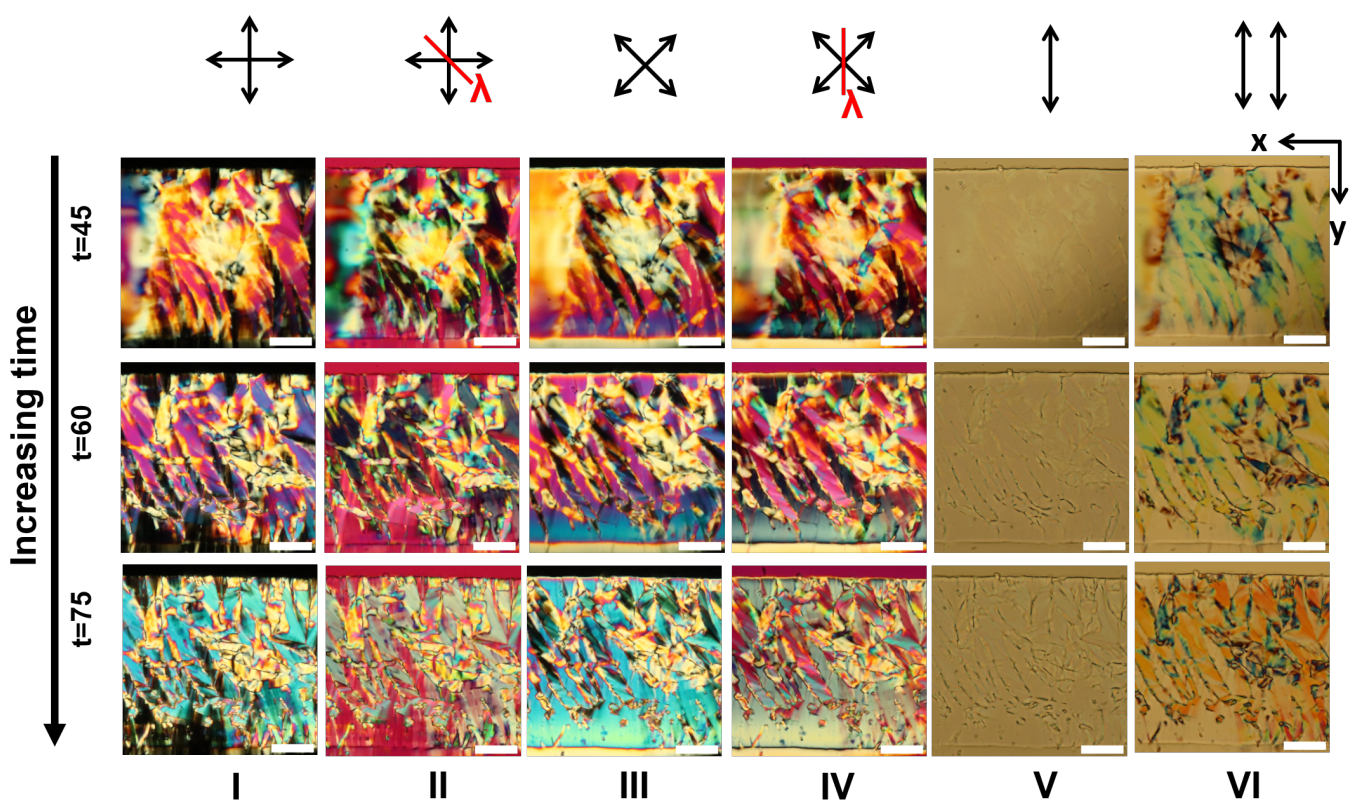

Figure A16. Degenerate planar anchoring within $200 \mu \mathrm{m}$ wide, $15 \mu \mathrm{m}$ deep microchannel yields M-phase textures at 14 wt. $\%$ DSCG. Imaging details are described previously. Here, time $(t)$ is in minutes after cooling ramp is concluded. (I-VI) shows the POM micrographs obtained from a combination of different polarizer orientations at the corresponding time. Scale bar: $50 \mu \mathrm{m}$. 


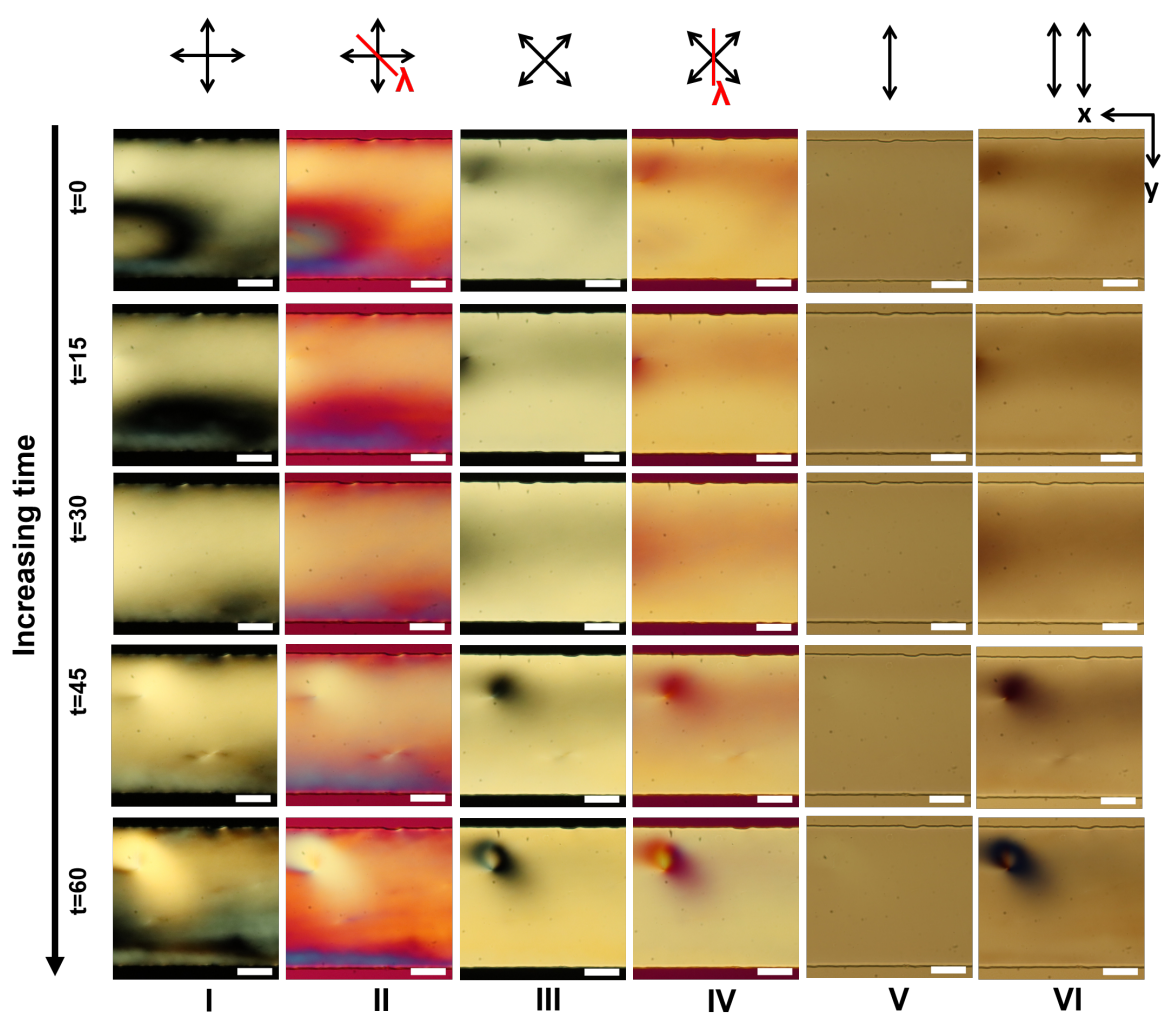

Figure A17. DSCG textures within $200 \mu \mathrm{m}$ wide, $15 \mu \mathrm{m}$ deep microchannel possessing homeotropic surface anchoring, here shown for $14 \mathrm{wt}$ \% DSCG. Imaging details are described previously. Here, time $(\mathrm{t})$ is in minutes after cooling ramp is concluded. (I-VI) shows the POM micrographs obtained from a combination of different polarizer orientations at the corresponding time. Scale bar: $50 \mu \mathrm{m}$.

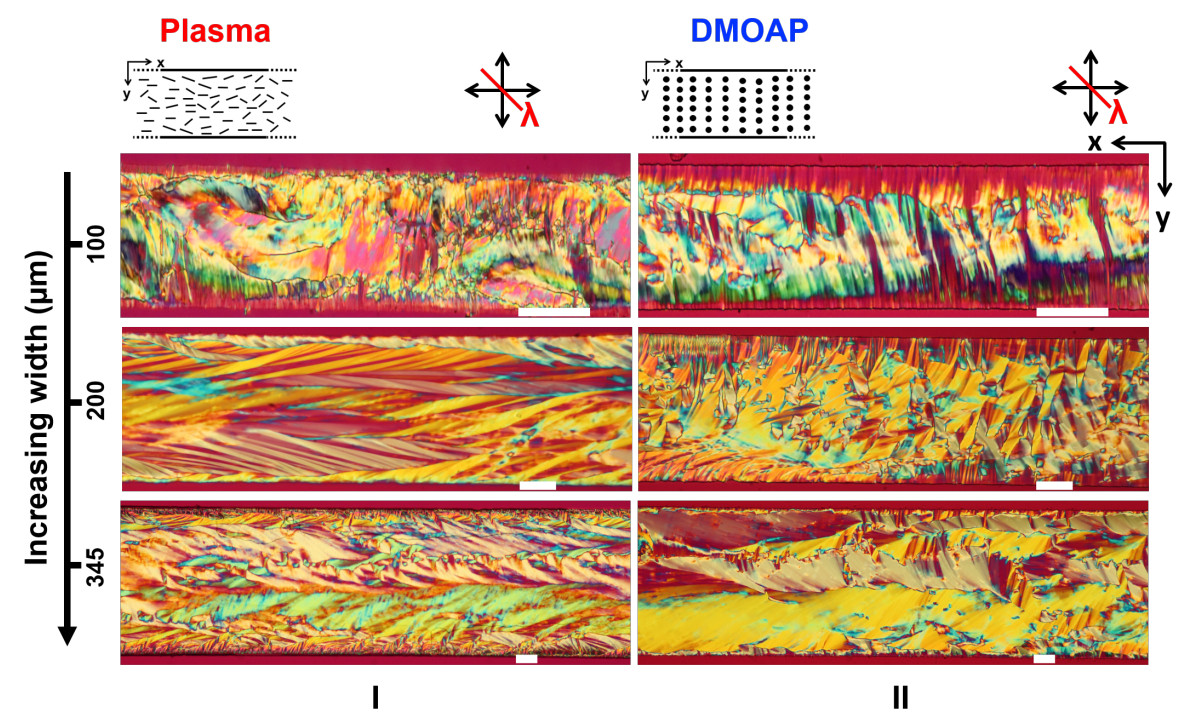

Figure A18. Herringbone and spherulite microfluidic textures formed by DSCG under degenerate planar and homeotropic anchoring conditions respectively. The textures corresponding to DSCG M-phase were observed between crossed polarizers with $\lambda$-retardation plate. The textures depend on the channel aspect ratio and imposed surface anchoring. (I) Microchannel with degenerate planar anchoring with dimensions: $(100 \mu \mathrm{m}$ wide, $10 \mu \mathrm{m}$ deep $),(200 \mu \mathrm{m}$ wide, $10 \mu \mathrm{m}$ deep $)$ and $(345 \mu \mathrm{m}$ wide, $10 \mu \mathrm{m}$ deep), respectively. Note the structural orientation of the herringbone texture along the channel length. (II) Observation of the spherulite texture in microchannels possessing homeotropic anchoring. The spherulite texture exhibits an overall orientation along the channel transverse direction (wall-to-wall). Scale bar: $50 \mu \mathrm{m}$. 


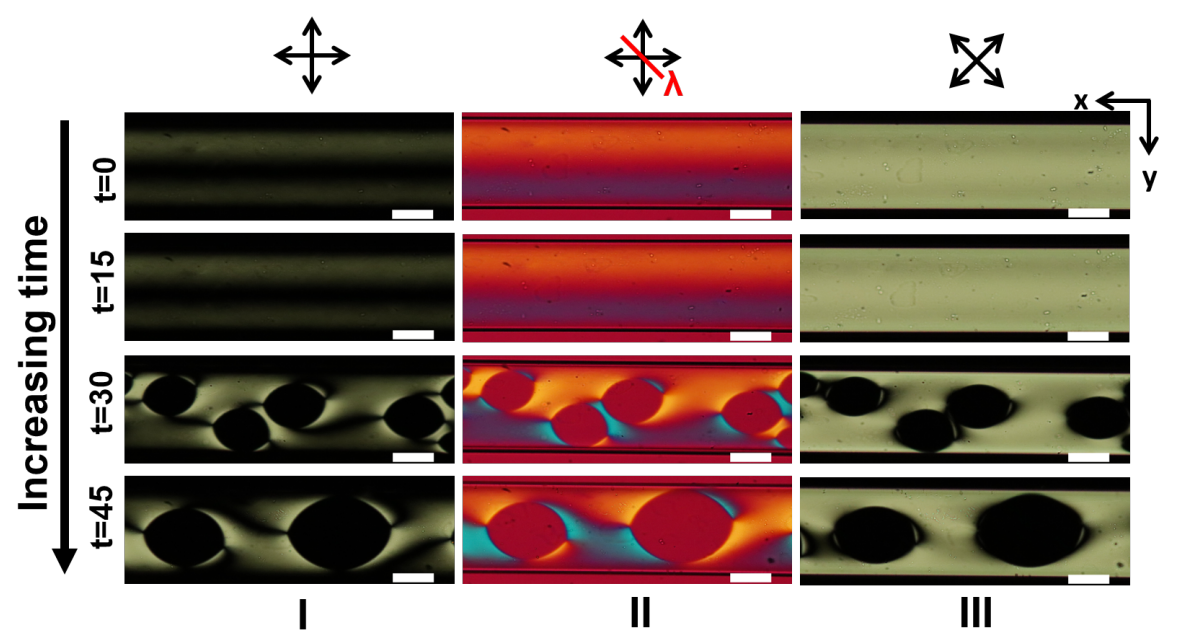

Figure A19. DSCG textures within untreated glass capillaries having weakly homeotropic anchoring. Evolution of textures exhibited by 14 wt.\% DSCG in $10 \times 100 \mu \mathrm{m}$ (depth $\times$ width) glass capillary as a function of time. Polarizing optical micrographs show the top view of the channel from $\mathrm{t}=0$ to $45 \mathrm{~min}$ : (I) between crossed polarizers; (II) crossed polarizers with $\lambda$-plate and (III) crossed polarizers at $45^{\circ}$ relative to channel length. Here, time (t) is in minutes after cooling ramp is concluded. No textural transition was observed, and the texture stabilizes to a typical homeotropic appearance (dark appearance between crossed polarizers, independent of the polarizer orientations. Scale bar: $50 \mu \mathrm{m}$.
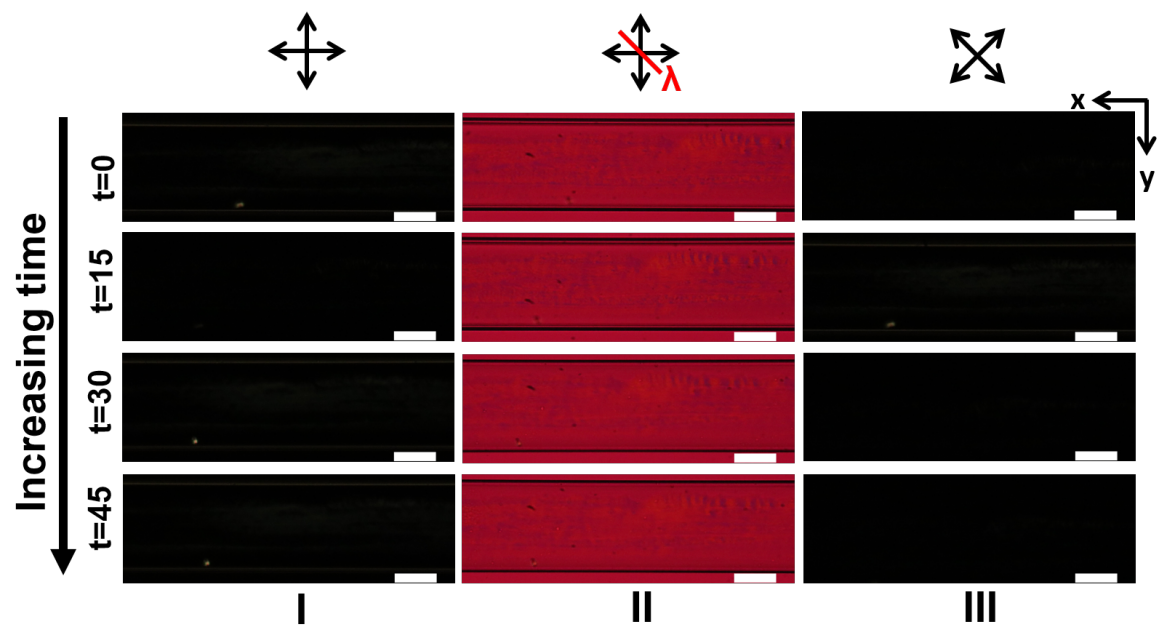

Figure A20. DSCG textures within DMOAP treated glass capillaries (strong homeotropic anchoring). The texture exhibited by $14 \mathrm{wt}$. $\%$ DSCG in $10 \times 100 \mu \mathrm{m}$ (depth $\times$ width) DMOAP treated glass capillary is stable over time. The POM textures confirm the homeotropic anchoring due to complete extinction of the polarized light (dark appearance). Here, time ( $t$ ) is in minutes after cooling ramp is concluded. (I-III) shows the POM micrographs obtained from a combination of different polarizer orientations at the corresponding time. Scale bar: $50 \mu \mathrm{m}$. 


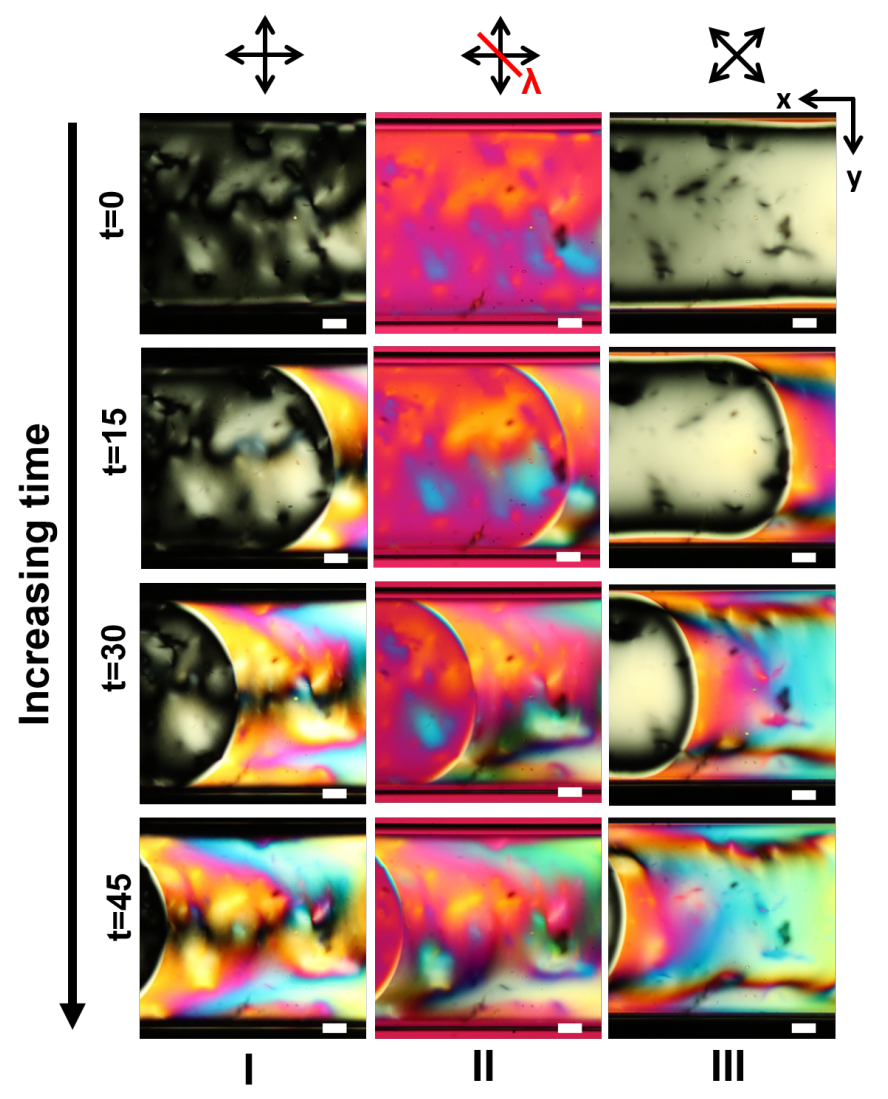

Figure A21. Evolution of DSCG textures (14 wt.\% DSCG) in $40 \mu \mathrm{m} \times 400 \mu \mathrm{m}$ untreated glass capillary, open from both sides. The transition from nematic to M-phase could be reproduced by allowing evaporation of water from the original DSCG solution (14 wt.\%). The sequence of images follow similar polarization configuration as described throughout the text. Here, time (t) is in minutes after cooling ramp is concluded. (I-III) shows the POM micrographs obtained from a combination of different polarizer orientations at the corresponding time. Scale bar: $100 \mu \mathrm{m}$.
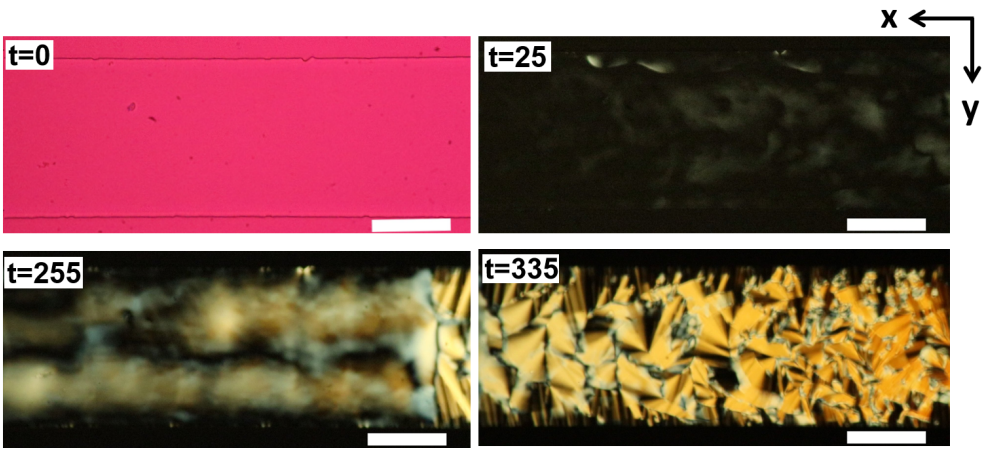

Figure A22. POM time lapse micrographs capture the isotropic-nematic-M phase transition of 12 wt. $\%$ DSCG within $200 \mu \mathrm{m}$ wide, $10 \mu \mathrm{m}$ deep microchannel with degenerate planar anchoring. Clockwise from top-left panel: isotropic phase observed under crossed polarizers with $\lambda$-plate inserted $(\mathrm{t}=0 \mathrm{~min})$; top-right panel shows crossed polarized micrograph at $\mathrm{t}=25 \mathrm{~min}$ : faint birefringence is noted, indicating nematic phase; bottom-right panel presents crossed polarized image at $t=255 \mathrm{~min}$, capturing the appearance of the $\mathrm{M}$-phase (right edge of the micrograph); and bottom-left panel at $\mathrm{t}=355 \mathrm{~min}$ shows the crossed polarized image of the spherulite (M-phase) texture. Here, time $(t)$ is in minutes after cooling ramp is concluded. Scale bar: $100 \mu \mathrm{m}$.

\section{References}

1. Lydon, J. Chromonic review. J. Mater. Chem. 2010, 20, 10071-10099. [CrossRef]

2. Lydon, J. Chromonic liquid crystalline phases. Liq. Cryst. 2011, 38, 1663-1681. [CrossRef] 
3. Zhou, S. Recent progresses in lyotropic chromonic liquid crystal research: elasticity, viscosity, defect structures, and living liquid crystals. Liq. Cryst. Today 2018, 27, 91-108. [CrossRef]

4. Lubensky, T.C. Confined chromonics and viral membranes. Mol. Cryst. Liq. Cryst. 2017, 646, 235-241. [CrossRef]

5. Hartshorne, N.H.; Woodard, G.D. Mesomorphism in the system disodium cromoglycate-water. Mol. Cryst. Liq. Cryst. 1973, 23, 343-368. [CrossRef]

6. Cox, J.S.; Woodard, G.D.; McCrone, W.C. Solid-state chemistry of cromolyn sodium (disodium cromoglycate). J. Pharm. Sci. 1971, 60, 1458-1465. [CrossRef]

7. Agra-Kooijman, D.M.; Singh, G.; Lorenz, A.; Collings, P.J.; Kitzerow, H.S.; Kumar, S. Columnar molecular aggregation in the aqueous solutions of disodium cromoglycate. Phys. Rev. E Stat. Nonlinear Soft Matter Phys. 2014, 89, 062504. [CrossRef]

8. Simon, K.A.; Sejwal, P.; Falcone, E.R.; Burton, E.A.; Yang, S.; Prashar, D.; Bandyopadhyay, D.; Narasimhan, S.K.; Varghese, N.; Gobalasingham, N.S.; et al. Noncovalent polymerization and assembly in water promoted by thermodynamic incompatibility. J. Phys. Chem. B 2010, 114, 10357-10367. [CrossRef]

9. Shiyanovskii, S.V.; Lavrentovich, O.D.; Schneider, T.; Ishikawa, T.; Smalyukh, I.I.; Woolverton, C.J.; Niehaus, G.D.; Doane, K.J. Lyotropic chromonic liquid crystals for biological sensing applications. Mol. Cryst. Liq. Cryst. 2005, 434, 259/[587]-270/[598]. [CrossRef]

10. Guo, F.; Mukhopadhyay, A.; Sheldon, B.W.; Hurt, R.H. Vertically Aligned Graphene Layer Arrays from Chromonic Liquid Crystal Precursors. Adv. Mater. 2011, 23, 508-513. [CrossRef] [PubMed]

11. Mushenheim, P.C.; Trivedi, R.R.; Tuson, H.H.; Weibel, D.B.; Abbott, N.L. Dynamic self-assembly of motile bacteria in liquid crystals. Soft Matter 2014, 10, 88-95. [CrossRef] [PubMed]

12. Zhou, S.; Sokolov, A.; Lavrentovich, O.D.; Aranson, I.S. Living liquid crystals. Proc. Natl. Acad. Sci. USA 2014, 111, 1265-1270. [CrossRef] [PubMed]

13. Collings, P.J.; van der Asdonk, P.; Martinez, A.; Tortora, L.; Kouwer, P.H. Anchoring strength measurements of a lyotropic chromonic liquid crystal on rubbed polyimide surfaces. Liq. Cryst. 2017, 44, 1165-1172. [CrossRef]

14. Lee, H.; Labes, M.M. Lyotropic cholesteric and nematic phaes of disodium cromoglycate in magnetic fields. Mol. Cryst. Liq. Cryst. 1982, 84, 137-157. [CrossRef]

15. Tone, C.M.; De Santo, M.P.; Buonomenna, M.G.; Golemme, G.; Ciuchi, F. Dynamical homeotropic and planar alignments of chromonic liquid crystals. Soft Matter 2012, 8, 8478-8482. [CrossRef]

16. Simon, K.A.; Burton, E.A.; Cheng, F.; Varghese, N.; Falcone, E.R.; Wu, L.; Luk, Y.Y. Controlling thread assemblies of pharmaceutical compounds in liquid crystal phase by using functionalized nanotopography. Chem. Mater. 2010, 22, 2434-2441. [CrossRef]

17. Jeong, J.; Han, G.; Johnson, A.T.C.; Collings, P.J.; Lubensky, T.C.; Yodh, A.G. Homeotropic Alignment of Lyotropic Chromonic Liquid Crystals Using Noncovalent Interactions. Langmuir 2014, 30, 2914-2920. [CrossRef]

18. Kim, J.Y.; Nayani, K.; Jeong, H.S.; Jeon, H.J.; Yoo, H.W.; Lee, E.H.; Park, J.O.; Srinivasarao, M.; Jung, H.T. Macroscopic alignment of chromonic liquid crystals using patterned substrates. Phys. Chem. Chem. Phys. 2016, 18, 10362-10366. [CrossRef]

19. Peng, C.; Guo, Y.; Turiv, T.; Jiang, M.; Wei, Q.H.; Lavrentovich, O.D. Patterning of Lyotropic Chromonic Liquid Crystals by Photoalignment with Photonic Metamasks. Adv. Mater. 2017, 29, 1606112. [CrossRef]

20. Dhakal, N.P.; Jiang, J.; Guo, Y.; Peng, C. Self-Assembly of Aqueous Soft Matter Patterned by Liquid-Crystal Polymer Networks for Controlling the Dynamics of Bacteria. ACS Appl. Mater. Interfaces 2020, 12, 13680-13685. [CrossRef]

21. Peng, C.; Turiv, T.; Guo, Y.; Wei, Q.H.; Lavrentovich, O.D. Command of active matter by topological defects and patterns. Science 2016, 354, 882-885. [CrossRef] [PubMed]

22. Turiv, T.; Koizumi, R.; Thijssen, K.; Genkin, M.M.; Yu, H.; Peng, C.; Wei, Q.H.; Yeomans, J.M.; Aranson, I.S.; Doostmohammadi, A.; et al. Polar jets of swimming bacteria condensed by a patterned liquid crystal. Nat. Phys. 2020, 16, 481-487. [CrossRef]

23. Nazarenko, V.G.; Boiko, O.P.; Park, H.S.; Brodyn, O.M.; Omelchenko, M.M.; Tortora, L.; Nastishin, Y.A.; Lavrentovich, O.D. Surface alignment and anchoring transitions in nematic lyotropic chromonic liquid crystal. Phys. Rev. Lett. 2010, 105, 017801. [CrossRef]

24. Tone, C.M.; De Santo, M.P.; Ciuchi, F. Alignment of chromonic liquid crystals: A difficult task. In Molecular Crystals and Liquid Crystals; Taylor \& Francis Group: Abingdon, UK, 2013; Volume 576, pp. 2-7. [CrossRef]

25. Jeong, J.; Kang, L.; Davidson, Z.S.; Collings, P.J.; Lubensky, T.C.; Yodh, A.G. Chiral structures from achiral liquid crystals in cylindrical capillaries. Proc. Natl. Acad. Sci. USA 2015, 112, E1837-E1884. [CrossRef]

26. Guo, Y.; Shahsavan, H.; Davidson, Z.S.; Sitti, M. Precise Control of Lyotropic Chromonic Liquid Crystal Alignment through Surface Topography. ACS Appl. Mater. Interfaces 2019, 11, 36110-36117. [CrossRef]

27. Zhou, S.; Nastishin, Y.A.; Omelchenko, M.M.; Tortora, L.; Nazarenko, V.G.; Boiko, O.P.; Ostapenko, T.; Hu, T.; Almasan, C.C.; Sprunt, S.N.; et al. Elasticity of lyotropic chromonic liquid crystals probed by director reorientation in a magnetic field. Phys. Rev. Lett. 2012, 109, 037801. [CrossRef]

28. Nayani, K.; Chang, R.; Fu, J.; Ellis, P.W.; Fernandez-Nieves, A.; Park, J.O.; Srinivasarao, M. Spontaneous emergence of chirality in achiral lyotropic chromonic liquid crystals confined to cylinders. Nat. Commun. 2015, 6, 8067. [CrossRef]

29. Tortora, L.; Lavrentovich, O.D. Chiral symmetry breaking by spatial confinement in tactoidal droplets of lyotropic chromonic liquid crystals. Proc. Natl. Acad. Sci. USA 2011, 108, 5163-5168. [CrossRef] 
30. Davidson, Z.S.; Kang, L.; Jeong, J.; Still, T.; Collings, P.J.; Lubensky, T.C.; Yodh, A.G. Chiral structures and defects of lyotropic chromonic liquid crystals induced by saddle-splay elasticity. Phys. Rev. E Stat. Nonlinear Soft Matter Phys. 2015, $91,050501$. [CrossRef]

31. Jeong, J.; Davidson, Z.S.; Collings, P.J.; Lubensky, T.C.; Yodh, A.G. Chiral symmetry breaking and surface faceting in chromonic liquid crystal droplets with giant elastic anisotropy. Proc. Natl. Acad. Sci. USA 2014, 111, 1742-1747. [CrossRef]

32. Nayani, K.; Fu, J.; Chang, R.; Park, J.O.; Srinivasarao, M. Using chiral tactoids as optical probes to study the aggregation behavior of chromonics. Proc. Natl. Acad. Sci. USA 2017, 114, 3826-3831. [CrossRef] [PubMed]

33. DIetrich, C.F.; Rudquist, P.; Lorenz, K.; Giesselmann, F. Chiral structures from achiral micellar lyotropic liquid crystals under capillary confinement. Langmuir 2017, 33, 5852-5862. [CrossRef] [PubMed]

34. Baza, H.; Turiv, T.; Li, B.X.; Li, R.; Yavitt, B.M.; Fukuto, M.; Lavrentovich, O.D. Shear-induced polydomain structures of nematic lyotropic chromonic liquid crystal disodium cromoglycate. Soft Matter 2020, 16, 8565-8576. [CrossRef] [PubMed]

35. Whitesides, G.M. The origins and the future of microfluidics. Nature 2006, 442, 368-373. [CrossRef]

36. Sengupta, A.; Tkalec, U.; Bahr, C. Nematic textures in microfluidic environment. Soft Matter 2011, 7, 6542-6549. [CrossRef]

37. Sengupta, A.; Pieper, C.; Enderlein, J.; Bahr, C.; Herminghaus, S. Flow of a nematogen past a cylindrical micro-pillar. Soft Matter 2013, 9, 1937-1946. [CrossRef]

38. Sengupta, A. Topological microfluidics: present and prospects. Liq. Cryst. Today 2015, 24, 70-80. [CrossRef]

39. Emeršič, T.; Zhang, R.; Kos, Z.; Čopar, S.; Osterman, N.; de Pablo, J.J.; Tkalec, U. Sculpting stable structures in pure liquids. Sci. Adv. 2019, 5, eaav4283. [CrossRef]

40. Sengupta, A.; Schulz, B.; Ouskova, E.; Bahr, C. Functionalization of microfluidic devices for investigation of liquid crystal flows. Microfluid. Nanofluidics 2012, 13, 941-955. [CrossRef]

41. Sengupta, A.; Tkalec, U.; Ravnik, M.; Yeomans, J.M.; Bahr, C.; Herminghaus, S. Liquid crystal microfluidics for tunable flow shaping. Phys. Rev. Lett. 2013, 110, 048303. [CrossRef]

42. Stieger, T.; Agha, H.; Schoen, M.; Mazza, M.G.; Sengupta, A. Hydrodynamic cavitation in Stokes flow of anisotropic fluids. Nat. Commun. 2017, 8, 15550. [CrossRef] [PubMed]

43. Giomi, L.; Kos, Z.; Ravnik, M.; Sengupta, A. Cross-talk between topological defects in different fields revealed by nematic microfluidics. Proc. Natl. Acad. Sci. USA 2017, 114, E5771-E5777. [CrossRef] [PubMed]

44. Sengupta, A.; Bahr, C.; Herminghaus, S. Topological microfluidics for flexible micro-cargo concepts. Soft Matter 2013, 9, 7251-7260. [CrossRef]

45. Na, Y.J.; Yoon, T.Y.; Park, S.; Lee, B.; Lee, S.D. Electrically Programmable Nematofluidics with a High Level of Selectivity in a Hierarchically Branched Architecture. ChemPhysChem 2010, 11, 101-104. [CrossRef] [PubMed]

46. Cuennet, J.G.; Vasdekis, A.E.; De Sio, L.; Psaltis, D. Optofluidic modulator based on peristaltic nematogen microflows. Nat. Photonics 2011, 5, 234-238. [CrossRef]

47. Cuennet, J.G.; Vasdekis, A.E.; Psaltis, D. Optofluidic-tunable color filters and spectroscopy based on liquid-crystal microflows. Lab Chip 2013, 13, 2721-2726. [CrossRef]

48. Wee, D.; Hwang, S.H.; Song, Y.S.; Youn, J.R. Tunable optofluidic birefringent lens. Soft Matter 2016, 12, 3868-3876. [CrossRef]

49. Sengupta, A. Tuning Fluidic Resistance via Liquid Crystal Microfluidics. Int. J. Mol. Sci. 2013, 14, 22826-22844. [CrossRef]

50. Wiese, O.; Marenduzzo, D.; Henrich, O. Microfluidic flow of cholesteric liquid crystals. Soft Matter 2016, 12, 9223-9237. [CrossRef]

51. Guo, Y.; Afghah, S.; Xiang, J.; Lavrentovich, O.D.; Selinger, R.L.; Wei, Q.H. Cholesteric liquid crystals in rectangular microchannels: Skyrmions and stripes. Soft Matter 2016, 12, 6312-6320. [CrossRef]

52. Sengupta, A.; Herminghaus, S.; Bahr, C. Liquid crystal microfluidics: Surface, elastic and viscous interactions at microscales. Liq. Cryst. Rev. 2014, 2, 73-110. [CrossRef]

53. Čopar, S.; Kos, Z.; Emeršič, T.; Tkalec, U. Microfluidic control over topological states in channel-confined nematic flows. Nat. Commun. 2020, 11, 59. [CrossRef] [PubMed]

54. Zimmermann, N.; Jünnemann-Held, G.; Collings, P.J.; Kitzerow, H.S. Self-organized assemblies of colloidal particles obtained from an aligned chromonic liquid crystal dispersion. Soft Matter 2015, 11, 1547-1553. [CrossRef] [PubMed]

55. Sharma, A.; Ong, I.; Mazza, M.; Sengupta, A. Surface-mediated stability of lyotropic textures in microfluidic environments. 2020, in prepration.

56. Sengupta, A.; Herminghaus, S.; Bahr, C. Nematic liquid crystals and nematic colloids in microfluidic environment. Mol. Cryst. Liq. Cryst. 2011, 547, 203-212. [CrossRef]

57. Sengupta, A. Topological Microfluidics: Nematic Liquid Crystals and Nematic Colloids in Microfluidic Environment; Springer International Publishing: Cham, Switzerland, 2013; Volume 1, pp. 1-150. [CrossRef]

58. Nastishin, Y.A.; Liu, H.; Schneider, T.; Nazarenko, V.; Vasyuta, R.; Shiyanovskii, S.V.; Lavrentovich, O.D. Optical characterization of the nematic lyotropic chromonic liquid crystals: Light absorption, birefringence, and scalar order parameter. Phys. Rev. E Stat. Nonlinear Soft Matter Phys. 2005, 72, 041711. [CrossRef]

59. Mirri, G.; Jampani, V.S.; Cordoyiannis, G.; Umek, P.; Kouwer, P.H.; Muševič, I. Stabilisation of 2D colloidal assemblies by polymerisation of liquid crystalline matrices for photonic applications. Soft Matter 2014, 10, 5797-5803. [CrossRef]

60. Bodas, D.; Khan-Malek, C. Hydrophilization and hydrophobic recovery of PDMS by oxygen plasma and chemical treatment-An SEM investigation. Sens. Actuators B Chem. 2007, 123, 368-373. [CrossRef] 
61. Mata, A.; Fleischman, A.J.; Roy, S. Characterization of Polydimethylsiloxane (PDMS) Properties for Biomedical Micro/Nanosystems. Biomed. Microdevices 2005, 7, 281-293. [CrossRef]

62. Bhattacharya, S.; Datta, A.; Berg, J.M.; Gangopadhyay, S. Studies on surface wettability of poly(dimethyl) siloxane (PDMS) and glass under oxygen-plasma treatment and correlation with bond strength. J. Microelectromech. Syst. 2005, 14, 590-597. [CrossRef]

63. Hillborg, H.; Ankner, J.F.; Gedde, U.W.; Smith, G.D.; Yasuda, H.K.; Wikström, K. Crosslinked polydimethylsiloxane exposed to oxygen plasma studied by neutron reflectometry and other surface specific techniques. Polymer 2000, 41, 6851-6863. [CrossRef]

64. Ruben, B.; Elisa, M.; Leandro, L.; Victor, M.; Gloria, G.; Marina, S.; Mian, S.K.; Pandiyan, R.; Nadhira, L. Oxygen plasma treatments of polydimethylsiloxane surfaces: Effect of the atomic oxygen on capillary flow in the microchannels. Micro Nano Lett. 2017, 12, 754-757. [CrossRef]

65. Toepke, M.W.; Beebe, D.J. PDMS absorption of small molecules and consequences in microfluidic applications. Lab Chip 2006, 6 , 1484-1486. [CrossRef] [PubMed]

66. Waters, L.J.; Finch, C.V.; Bhuiyan, A.K.H.; Hemming, K.; Mitchell, J.C. Effect of plasma surface treatment of poly(dimethylsiloxane) on the permeation of pharmaceutical compounds. J. Pharm. Anal. 2017, 7, 338-342. [CrossRef] [PubMed]

67. Yoshimi, A.; Hashizume, H.; Tamaki, S.; Tsuda, H.; Fukata, F.; Nishimura, K.i.; Yata, N. Importance of Hydrolysis of Amino Acid Moiety in Water-Soluble Prodrugs of Disodium Cromoglycate for Increased Oral Bioavailability. J. Pharmacobio Dyn. 1992, 15, 339-345. [CrossRef] [PubMed] 